\title{
Nonlocal effects in the plasmons of nanowires and nanocavities excited by fast electron beams
}

\author{
Javier Aizpurua* \\ Donostia International Physics Center (DIPC) and Centro Mixto de Física de Materiales CSIC-UPV/EHU, Paseo Manuel Lardizabal 4, \\ Donostia-San Sebastián 20018, Spain \\ Alberto Rivacoba \\ Department of Materials Physics, University of the Basque Country (UPV/EHU) and Centro Mixto de Física de Materiales \\ CSIC-UPV/EHU, Apartado 1072, Donostia-San Sebastián 20080, Spain
}

(Received 12 March 2008; published 2 July 2008)

\begin{abstract}
The solutions of surface modes in cylindrical metallic wires and cavities are obtained within a nonlocal dielectric formalism. We compare the results with those obtained from standard local approaches. The specular reflection model is applied to describe the nonlocal potentials in the vicinity of the wires and cavities. The external probe exciting the surface plasmons are fast electron beams traveling parallel to the wires and cavities, as those commonly used in electron microscopy and cathodoluminescence. Energy-loss spectra due to surfaceplasmon excitation are calculated with use of the nonlocal formalism both for electron trajectories near a metallic nanowire and a metallic nanocavity. When nonlocal effects are considered, the intensity of the plasmon excitation is reduced, and a blueshift of the energy is observed. This effect is more pronounced for very thin wires and cavities where the cylindrical interfaces are strongly interacting. The blueshifts reported here are important for the accurate design of the plasmon response in one-dimensional metallic nanostructures.
\end{abstract}

DOI: 10.1103/PhysRevB.78.035404

PACS number(s): 73.20.Mf, 79.20.Uv, 68.37.Lp, 78.70.-g

\section{INTRODUCTION}

The role of surface plasmons is central in the interaction of charged probes and radiation with metallic structures. Since Ritchie ${ }^{1}$ predicted the existence of these low-energy excitations 50 years ago, there has been a continuous effort to characterize and engineer the plasmon response in a variety of configurations. Typically, the properties of plasmon excitations depend on the nature of the probe or exciting field, on the shape of the target, on its size, and on the couplings established with neighboring targets and environment. ${ }^{2}$ Experimental evidence of the excitation of surface plasmons by charged probes dates back to the experiments by Powell and Swan, ${ }^{3}$ extracted from the analysis of electron-energy-loss spectroscopy (EELS) in metallic interfaces. Even though the current interest in surface plasmons is commonly associated with nano-optics due to the broad set of applications in spectroscopy, sensing, communications, and biomedical applications, fast electrons can be an excellent complementary probe for exciting certain modes which are not available optically. ${ }^{4}$ In that spirit, in cathodoluminescence, the combination of a fast electron probe with an optical detection scheme has recently made possible the spatial mapping of surface-plasmon modes in different metallic nanostructures. ${ }^{5-8}$ The development of the scanning transmission electron microscopy (STEM) over the years, where a well focused and highly energetic electron beam is used to obtain high-resolution energy-loss spectra $^{9}$ of valence electrons, has opened EELS to the study of the collective lowenergy excitations (surface plasmons) in a variety of configurations, such as in small particles, ${ }^{10-14}$ cylindrical cavities, ${ }^{15,16}$ nanowires, ${ }^{17-20}$ fullerenes, ${ }^{21-27}$ and carbon nanotubes. ${ }^{28-31}$ In many of these works, the inelastic interaction between the fast electrons and the target has been described in the framework of the classical dielectric theory. ${ }^{1,32-45}$ Within this approach, the collective excitations of a bulk material are described by a local dielectric function $\varepsilon(\omega)$ (usually measured optically), providing a detailed description of the valence electron energy-loss spectra (bulk modes of a few eV in energy). The consideration of the geometrical boundaries in an inhomogeneous dielectric configuration accounts for the presence of surface modes, giving rise to surface losses.

In this work we focus on the plasmon excitation by an electron beam in metallic nanowires and nanocavities, as schematically shown in Fig. 1. After the calculations of the stopping power of fast probes in a particle accelerator, ${ }^{46}$ the first works on the interaction between charged probes and cylindrical interfaces were motivated by the ability of electrons in STEM to drill holes on films, creating cylindrical cavities $^{15,16}$ (see Fig. 1). The application of EELS to study

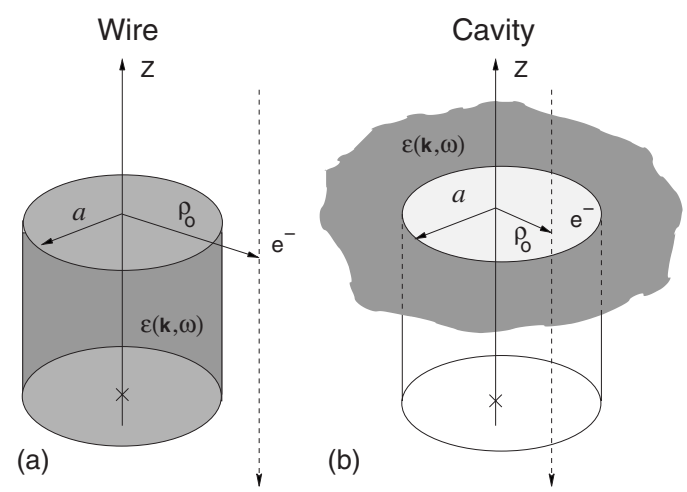

FIG. 1. (a) Schematics of a cylindrical metallic nanowire of radius $a$ characterized by a nonlocal dielectric function $\varepsilon(\mathbf{k}, \omega)$ with an electron $e^{-}$passing parallel to the wire axis at an impact parameter $\rho_{0}$ in an external trajectory $\left(\rho_{0}>a\right)$. Internal trajectories $\left(\rho_{0}\right.$ $<a$ ) are also considered. (b) Cylindrical cavity in the same material with an electron passing along the cavity in an internal trajectory $\left(\rho_{0}<a\right)$. External trajectories $\left(\rho_{0}>a\right)$ are also considered. 
nanowires led to theoretical descriptions of energy losses experienced by electron probes in the proximity of more complex cylindrical structures and nanotubes. ${ }^{47-51}$ Aspects such as anisotropy in the response can also be considered within the dielectric approach and have been applied to describe, for example, energy losses in multishell fullerenes and carbon nanotubes. ${ }^{52,53}$ Retardation in the interaction has also been considered, ${ }^{16,54}$ resulting in a small effect for thin metallic wires. The ability of the electron beam to produce Cherenkov radiation in cylindrical cavities, an event occurring when the electron travels with velocity $v$ larger than the velocity of light in the surrounding medium, $c / \sqrt{\varepsilon}$, gives extra contributions to the loss spectrum ${ }^{55}$ and has provided further information about the target structure. ${ }^{56}$

The $k$ dependence of the nonlocal dielectric function $\varepsilon(\mathbf{k}, \omega)$, which is energy $\hbar \omega$ and also momentum $\mathbf{k}$ dependent, arises from the spatial correlation between the induced charge density, an effect which prevents the pilling up of electrons at short distances. In electron microscopy, there can be situations where the momentum transfer is considerable, and a local dielectric response is not enough to accurately describe the interaction between electron beam and sample. This is the case, for example, where two interfaces interact in very close proximity, or when large scattering angles of the incident beam are used, requiring higher values of the transferred momentum. In such a situation, one needs to consider a dispersive dielectric function to describe the medium. This situation can be also present in nano-optics, with the targets excited by light, in cases where the distances between interfaces are smaller that the electron spill-out distance. In that case, nonlocal corrections of the response should produce a difference between the surface mode positions and excitation strengths. Nonlocal effects have been largely studied in the planar geometry, for example, where different features of spatial dispersion have been considered to obtain the image potential outside a metallic semi-infinite planar interface ${ }^{57-60}$ In the context of the interaction with electron beams, previous works ${ }^{42,61}$ concluded that dispersive effects are important in the planar case when the electron beam travels closer than $0.5 \mathrm{~nm}$ to the interface. In the case of nanoparticles or nanowires, where the induced charge density is always limited into very small regions and therefore strongly interacting, this correlation is expected to be stronger. Therefore nonlocal effects are likely to be more relevant.

The consideration of nonlocality requires the use of additional boundary conditions for the surface charge densities. Here we present a study of nonlocality in a cylindrical geometry within the specular reflection model (SRM), ${ }^{57}$ also known as semiclassical infinite-barrier (SCIB) model. ${ }^{62}$ The most important feature of this model is that the electrons in a metal are assumed to form a uniform positive background (jellium) which terminates at the surface. The electron density is constant inside the metal and drops suddenly to zero outside the surface. The SRM has been used, for example, to describe nonlocality in spherical geometries. ${ }^{63-65}$ The SRM neglects an important feature of the surface of a metal: the electron density does not terminate suddenly at the edge of the jellium but spills out into the vacuum. For very small particles, if one takes the electron spillout into account, it is possible to find energies of the surface modes different from those presented here. ${ }^{66}$ This inadequacy of the SRM occurs mainly in the range where quantum size effects become important, i.e., for particles smaller than $\approx 1 \mathrm{~nm}$. In this case, the detailed results of the absorption peaks and the energy shifts will depend on the electron wave functions and the electron density near the surface ${ }^{67-70}$ Nevertheless, the SRM includes the main effect of nonlocality for particles larger than $\approx 1 \mathrm{~nm}$ and will allow us to study the effect of the nonlocal response in the dispersion of cylindrical modes in nanowires and nanocavities (Sec. II). In the limit of thin wires, the nonlocal modes obtained here can be related to the dispersion relation of a quasi-one-dimensional electron gas. ${ }^{71,72} \mathrm{We}$ also analyze the excitation of these modes by an electron beam when traveling in the proximity of both nanowires (Sec. III) and cavities (Sec. IV) to understand the energy shifts derived from the consideration of nonlocal effects. Dispersive effects in cylindrical nanostructures have been also studied by other authors in terms of the band structure of the cylinder. ${ }^{50,73,74}$ An attempt to extend the existing classical dielectric approach has been made ${ }^{75}$ by partially considering momentum transfer along the wire axis but not considering momentum transfer perpendicular to the wire, therefore missing the effects related to the electron density correlation. More sophisticated methods based on a selfconsistent solution of the electronic density within the timedependent density-functional theory (TDDFT) can give full account of nonlocal effects in the response. These methods are usually developed for standard geometries, ${ }^{68,76-80}$ but the computational requirements allow the tackling of only small systems with a limited number of electrons involved. Complex $a b$ initio calculations, fully accounting for the exchange and correlation of the electron density, can also address the existence of interactions between bulk and surface states that give rise to low-energy acoustic plasmons in twodimensional systems. ${ }^{81,82}$

Expressions for the nonlocal potentials within the SRM are derived in Appendix A for wires and in Appendix B for cavities. In the analytical expressions, atomic units have been used throughout, whereas in the analysis of the loss spectra we have favored the use of electron volts $(\mathrm{eV})$ and nanometers $(\mathrm{nm})$.

\section{MODES OF CYLINDRICAL NANOSTRUCTURES}

In an electrostatic approach, the surface modes in a metallic system are the set of frequencies for which Laplace equation features nontrivial solutions. In the visible range, these solutions represent collective excitation of the electron gas. In a nonbounded medium, the bulk-plasmon frequency presents a dispersion $\omega_{p}(k)$, where $\omega_{p}$ is the plasma frequency of the metal, which depends on the electron density $n$, charge $e$, and mass $m$, as $\omega_{p}=\sqrt{\frac{4 \pi n e^{2}}{m}}$. The energy of the bulk plasmon contains the wavelength dependence of the response of the electron gas, as a consequence of correlation effects in the induced charge density. In bounded systems, in addition to this $k$ dependence, there is another source of dispersion in the spectrum of modes, due to the coupling of the induced charge density at the surfaces of the system. These solutions are the so-called surface plasmons ${ }^{1,83}$ and can be 
detected, for example, in the energy-loss spectra of fast charged particles ${ }^{10,84}$ or in optical spectroscopy. ${ }^{2}$ Neglecting the $k$ dependence of the dielectric function, the modes of highly symmetric systems (planar interfaces, films, spheres, cylindrical tubes or cavities, coupled spheres, and spheroids) can be easily calculated by assuming a local dielectric function $\varepsilon(\omega)$ to characterize the bulk response of the material and by imposing the standard continuity conditions of the potential and of the normal component of the displacement at the interface. In the case of a momentum-dependent response function $\varepsilon(\mathbf{k}, \omega)$, one needs an additional boundary condition at the interface. As mentioned above, here we will use the $\mathrm{SRM},{ }^{57}$ applied in a cylindrical geometry to treat both nanowires and nanocavities (see the schematics in Fig. 1). The details of the nonlocal potentials for both wires and cavities are presented in Appendixes A and B, respectively.

To describe the $k$ dependence of the dielectric response, we will assume a dielectric function given by the plasmon pole approximation (PPA) ${ }^{85}$ which accounts for the freeelectron oscillations and partially for the electron-hole pair generation in the material:

$$
\varepsilon(k, \omega)=1-\frac{\omega_{p}^{2}}{\omega(\omega+i \gamma)-\beta^{2} k^{2}-\frac{1}{4} k^{4}},
$$

where $\omega_{p}$ is the plasma frequency, $\beta=\sqrt{3 / 5} v_{F}, v_{F}$ is the Fermi velocity of the electron gas, and $\gamma$ is the damping, which represents the inverse of the plasmon lifetime. Equation (1) is a useful approximation of the random-phase approximation (RPA): In the small $k$ limit it reproduces the bulk-plasmon dispersion relation, while in the large $k$ limit it tends to the energy-momentum relationship of a single electron, $\omega=k^{2} / 2$. This approximation has been used in threeand two-dimensional systems, with good agreement with more sophisticated treatment and experimental results. In the study of the excitations produced by fast electrons, where the momentum transfer is small, the results of this model do not differ significantly from results obtained with use of another nonlocal dielectric function, based on another assumption for the nonlocal dependence, such as the hydrodynamic model, for example. Nonlocal effects in materials commonly used in plasmonics such as gold and silver can also be addressed within this model with the use of an appropriate nonlocal response function.

\section{A. Nonlocal modes of a nanowire}

Let us consider an infinite metallic wire of radius $a$ with the axis oriented along the $z$ axis. The surface modes are the values of $\omega$, which are solutions of potentials when there is no external field. They correspond to the zeros of the denominator of the nonlocal potentials [see Eqs. (A17) and (A20) in Appendix A]:

$$
\widetilde{\varepsilon}_{m}\left(a, q_{z}, \omega\right) K_{m}^{2}\left(q_{z} a\right) I_{m}^{\prime}\left(q_{z} a\right)-K_{m}^{\prime}\left(q_{z} a\right)=0,
$$

where $I_{m}(x)$ and $K_{m}(x)$ are the modified Bessel functions of order $m .{ }^{86} f^{\prime}(x)$ stands for the derivative of the function $f$, and $q_{z}$ is the $z$ component of the wave vector $k$. The function $\widetilde{\varepsilon}_{m}\left(\rho, q_{z}, \omega\right)$ is defined for a given radial cylindrical component $\rho$ of the vector position as

$$
\frac{1}{\widetilde{\varepsilon}_{m}\left(\rho, q_{z}, \omega\right)}=\int_{0}^{\infty} \frac{J_{m}(Q \rho) J_{m}(Q a)}{\left[Q^{2}+q_{z}^{2}\right] \varepsilon(\mathbf{k}, \omega)} Q d Q,
$$

where $J_{m}(x)$ stands for the Bessel functions of first kind. ${ }^{86}$ The momentum $k$ in the dielectric function is $k=\sqrt{Q^{2}+q_{z}^{2}}$, with $Q$ as the radial component of the wave vector $\mathbf{k}$.

In the case of a local response, where $\varepsilon=\varepsilon(\omega)$, the integral in Eq. (3) can be analytically solved, ${ }^{87}$ obtaining

$$
\widetilde{\varepsilon}_{m}\left(\rho, q_{z}, \omega\right)= \begin{cases}\frac{\varepsilon(\omega)}{I_{m}\left(q_{z} \rho\right) K_{m}\left(q_{z} a\right)} & \text { if } \rho<a \\ \frac{\varepsilon(\omega)}{K_{m}\left(q_{z} \rho\right) I_{m}\left(q_{z} a\right)} & \text { if } \rho>a .\end{cases}
$$

In this case, Eq. (2) reduces to the local expression of the modes of a cylinder: ${ }^{47,88}$

$$
\varepsilon(\omega) I_{m}^{\prime}\left(q_{z} a\right) K_{m}\left(q_{z} a\right)-I_{m}\left(q_{z} a\right) K_{m}^{\prime}\left(q_{z} a\right)=0 .
$$

In a general case, the dispersion equation of modes [Eq. (2)] has to be numerically solved. Solutions of Eq. (2) are in general complex. The real part provides the energy of the mode, while its imaginary part stands for the inverse of the lifetime of the excitation. Here we have considered a real $\varepsilon(\mathbf{k}, \omega)$ function by taking $\gamma=0$ in Eq. (1). The energy of the $m$ th nonlocal mode depends on both the variables $a$ and $q_{z}$; i.e., $\hbar \omega_{m}\left(a, q_{z}\right)$ is not a function of the product $q_{z} a$ as in the local case. Figures 2(a) and 2(b) show the dependence of the dispersion of the $m=0$ and $m=1$ wire modes as a function of the parallel momentum $q_{z}$ for three different values of the cylinder radius $(a=1,3$, and $10 \mathrm{~nm})$. Results are displayed as solid lines for the modes obtained with use of a nonlocal dielectric function, whereas those obtained with use of a local response are displayed as dashed lines. Both modes show a blueshift (larger energy) of the nonlocal results with respect to their respective local results. The $m=0$ mode in Fig. 2(a) tends to zero for small values of $q_{z}$, consistent with experimental results of the dispersion of plasmon wires in atomic metallic chains. ${ }^{19}$ For large wires $(a=10 \mathrm{~nm}$, green lines), the blueshift of the nonlocal approach is noticeable only for large values of parallel momentum, whereas for smaller wires $(a=1 \mathrm{~nm}$, blue lines) the blueshift is already noticeable for small momentum values. The linear dispersion of the planar surface plasmon (black solid line) is recovered for large momentum components and large wire sizes. ${ }^{57}$ In Fig. 2(b) the $m=1$ modes are shown for the same sizes as in (a). Similar tendencies are found for the blueshifts in this case. Larger shifts occur for large momentum components and smaller wires. The limit of this mode for $a \rightarrow 0$ is the planar surface plasmon at $\omega_{p} / \sqrt{2}$ (solid line). In Figs. 2(c) and 2(d) we show contour plots of the difference in energy $(\mathrm{eV})$ between the modes in the nonlocal and local approaches for a set of wire sizes and parallel momentum components. As expected from Figs. 2(a) and 2(b), significant differences between the local and nonlocal responses appear for large momentum $q_{z}$ and for small values of the radius $a$ because the transversal component of the momentum associated with the $m$ th mode depends on the parameter $m / a$. This explains the fact that the local limit is recovered for low $m$ modes only for small values of $q_{z}$ and large radii. In all the cases, when 

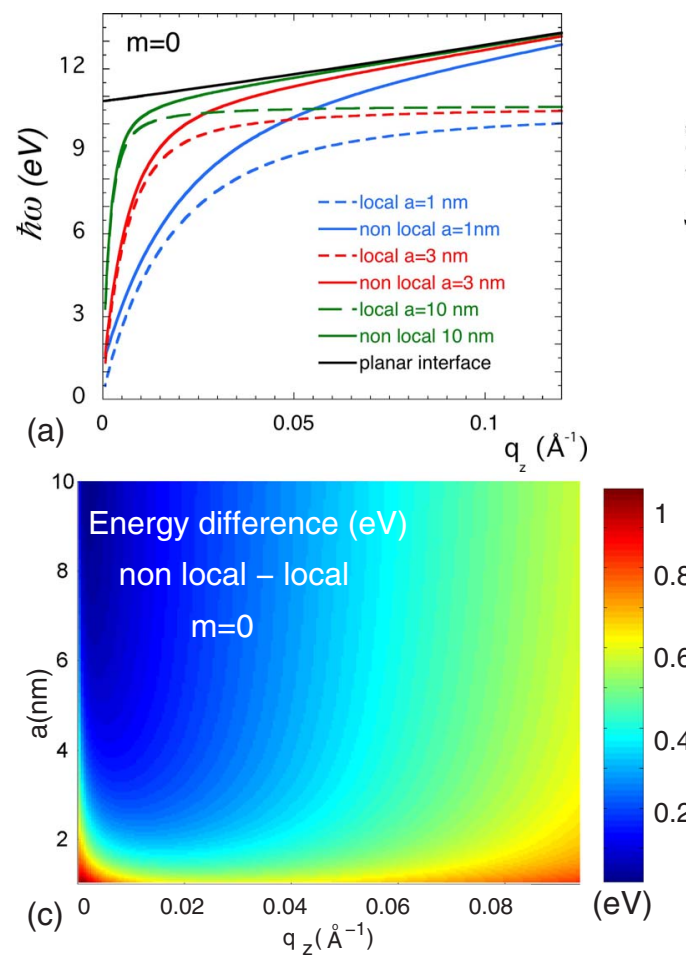
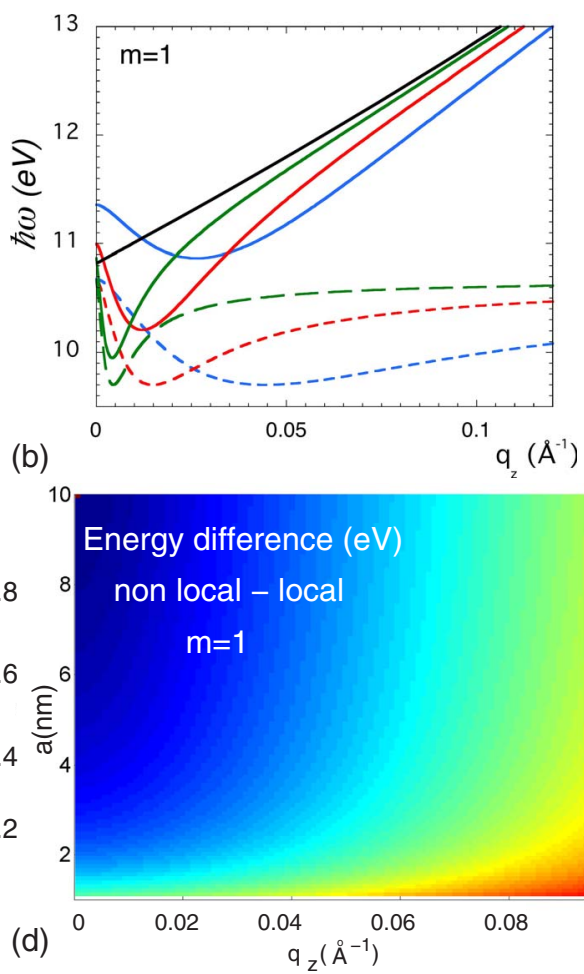

FIG. 2. (Color online) Energy (in eV) of the plasmon modes in three different wires of radii $a=1 \mathrm{~nm}$ (blue lines), $a=3 \mathrm{~nm}$ (red lines), and $a=10 \mathrm{~nm}$ (green lines) for azimuthal number (a) $m=0$ and (b) $m=1$ as a function of the parallel momentum component $q_{z}$. The modes within a nonlocal approach are displayed (solid lines) and compared to those within a local approach (dashed lines). The nonlocal dielectric function is given by the PPA in Eq. (1) for a metal with parameter $r_{s}=2.117$ a.u., giving a plasma frequency $\omega_{p}=15.3 \mathrm{eV}$ and $\beta$ $=0.7$ a.u. Zero damping is assumed in these calculations. The limit of the nonlocal planar surface plasmon is displayed as a solid black line. [(c) and (d)] Contour plots of the energy difference (in eV) between the nonlocal and the local wire plasmon mode solutions for different radii $a$ and momentum components $q_{z}$ for azimuthal numbers $m=0$ and $m=1$, respectively.

the radius becomes extremely small $(a \rightarrow 0)$, the spectrum of modes for a nanowire when nonlocality is considered differs largely from that obtained in the local approach. For $m \neq 0$, the frequencies of the nonlocal modes can exceed the bulkplasmon frequency $\omega_{p}$, while in the local approach, these modes gather together around the surface-plasmon frequency $\omega_{p} / \sqrt{2}$. This difference is more noticeable in the $m=0$ mode, where the energy of the nonlocal mode diverges as $a \rightarrow 0$ [red area showing an increased difference in Fig. 2(c)], while the energy of the local mode tends to the static limit $\omega_{0}=0$. This result is in agreement with the nonlocal response within the RPA of a one-dimensional electron gas, which shows the dependence of the frequency of the plasmon $\omega$ as $\omega$ $\propto q_{z} \ln \left(q_{z} a\right) .{ }^{71}$ This result is convergent for small $q_{z}$ values but diverges for small $a$. Consideration of retardation in the response lifts this divergence for small radius. ${ }^{72}$ The blueshifts obtained here for the wire plasmon modes are relevant and valid in most physical situations dealing with wire radius of nanometers or even angstroms. However, for subangstrom wires, the divergent result for $a \rightarrow 0$ should be considered not physical since the one-dimensional response is constructed based on a three-dimensional bulk response, which obviously cannot describe the response in the transversal direction correctly and therefore the one-dimensional nature of the wire with $a=0$.

To illustrate nonlocal effects in standard situations in plasmonics, we present in Fig. 3 the results for the wire modes as a function of the size of the wire (radius $a$ ) for the particular case of the modes $m=0$ [Fig. 3(a)] and $m=1$ [Fig. 3(b)]. The $m=0$ mode shown in (a) is typically excited by an electron probe traveling in a central trajectory with velocity $v$. The relevant momentum component $q_{z}$ in this case is given by $q_{z}=\omega / v$. As the size of the wires becomes smaller, the nonlocal (blue line) and the local (red line) approaches depart from each other, with the energy difference larger than $1 \mathrm{eV}$ for wires smaller than $4 \mathrm{~nm}$. The $m=1$ mode in a wire [Fig. 3(b)] can be excited by an electron beam traveling at a certain distance from the wire (solid lines) and also by an optical probe traveling parallel to the wire axis with wave vector $k=q_{z}$ given by $q_{z}=\omega / c$ (dashed lines). Local (red lines) and nonlocal modes (blue lines) are displayed. Similar to the $m$ $=0$ mode, the results show a clear blueshift in the response (difference between blue and red lines) for thin enough wires, which can be of up to several tenths of $\mathrm{eV}$ for wires as large as $10 \mathrm{~nm}$ wide. Therefore, the influence of nonlocal effects in the description of an accurate plasmon response has enormous implications in metallic nanosystems with highly interacting interfaces, as it is the case in thin wires. The dependence on nonlocal effects obtained here for wires is consistent with that presented for other highly interacting interfaces, such as thin films, ${ }^{1}$ small spheres, ${ }^{63}$ close dimers, ${ }^{64}$ or nanoshells, ${ }^{65}$ reporting an overall blueshift of the plasmon modes. 

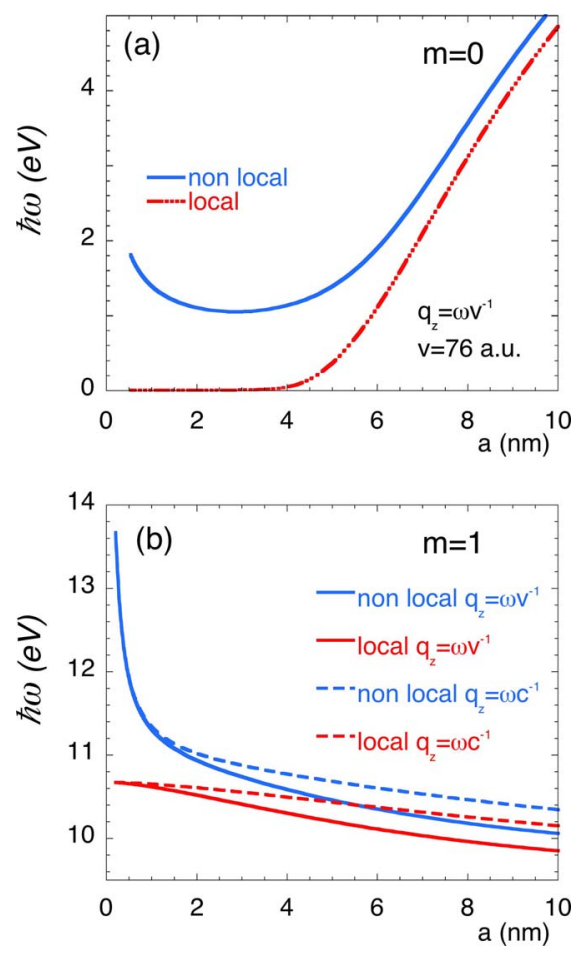

FIG. 3. (Color online) Energy of the wire plasmons as a function of the size of the wires for two typical situations of plasmon excitation. (a) $m=0$ mode as a function of wire radius $a$. This mode is commonly excited by an electron traveling parallel to the wire axis in a central trajectory. The relevant momentum component in this case is given by $q_{z}=\omega / v$. A $100 \mathrm{keV}$ electron beam corresponds to a velocity $v$ of 76 a.u. The blue line denotes the nonlocal correction, whereas the red line stands for the local approach. (b) $m=1$ mode commonly excited (i) by an optical plane wave with wave vector $\mathbf{k}$ and polarization transversal to the axis wire (dashed lines) or (ii) by an electron beam traveling outside the wire at a certain distance (solid lines). The momentum component of the incident plane wave in the optical excitation is given by $k=q_{z}=\omega / c$. The dielectric response is considered as in Fig. 2 and nonlocal (blue lines) and local (red lines) approaches are compared. Nonlocal effects produce a blueshift of several tenths of $\mathrm{eV}$ for wires with radius smaller than $4 \mathrm{~nm}$.

\section{B. Nonlocal modes of a cavity}

The modes of a cylindrical cavity of radius $a$ drilled in a metallic medium characterized by the nonlocal dielectric function $\varepsilon(\mathbf{k}, \omega)$ are given by the solutions of the following equation [zeros of the denominators of the potentials in Eqs. (B2) and (B3) in Appendix B]:

$$
\widetilde{\varepsilon}_{m}\left(a, q_{z}, \omega\right) I_{m}^{2}\left(q_{z} a\right) K_{m}^{\prime}\left(q_{z} a\right)-I_{m}^{\prime}\left(q_{z} a\right)=0 .
$$

In the local case, this expression reproduces the equation for the local modes:

$$
\varepsilon(\omega) I_{m}\left(q_{z} a\right) K_{m}^{\prime}\left(q_{z} a\right)-I_{m}^{\prime}\left(q_{z} a\right) K_{m}\left(q_{z} a\right)=0 .
$$

In Figs. 4(a) and 4(b), we plot the energy of the $m=0$ and $m=1$ modes as a function of the parallel momentum component $q_{z}$ for three different cavities with the radii as in Figs. 2(a) and 2(b). Contour plots showing the difference in energy between the nonlocal and local approaches are shown in Fig. 4(c) $(m=0)$ and Fig. 4(d) $(m=1)$. The departure of the nonlocal modes from the local mode is qualitatively similar to that found for wires. The energies of the nonlocal approach [solid lines in Figs. 4(a) and 4(b)] are similar to the local ones (dashed lines) only for very small values of $q_{z}$ and for wide cylinders [blue areas in the contour plots of Figs. 4(c) and 4(d)]. In the case of small momentum $q_{z} \rightarrow 0$ and large cavity size $a \gg 10 \mathrm{~nm}$, the modes tend to the value of the bulk-plasmon energy $\hbar \omega_{p}$ for $m=0$ and to energies close to the planar surface-plasmon energy $\hbar \omega_{p} / \sqrt{2}$ for $m=1$.

Both in cavities and in wires, the relevance of the nonlocal corrections to the energy-loss spectrum in electron microscopy depends not only on the radius of the structure but also on the range of the $q_{z}$ values involved in the excitation spectrum, strongly dependent on the electron beam trajectory. In the case of a fast electron beam with velocity $v$ parallel to the cylinder axis, the value $q_{z}$ involved in the excitation spectrum is $q_{z}=\omega / v$, typically $\sim 0.01 \AA^{-1}$. Therefore in this situation, for realistically small cylinders ( $a$ $\sim 2-5 \mathrm{~nm}$ ), where the main contribution to the inelastic scattering arises from the coupling to the first $m$ modes, the nonlocal corrections to the position of the energy of the plasmon peaks in EELS are expected to be small. The nonlocal correction is likely to be large in the case of trajectories nonparallel to the axis, a situation more suitable experimentally in the study of nanowires. Zabala et al. ${ }^{49}$ showed that in the local approach for a nanowire of radius $a=2 \mathrm{~nm}$, the surface peak arising from the $m \geq 1$ modes has a nonnegligible contribution to the loss spectrum of values of $q_{z}$ up to $0.18 \AA^{-1}$. The result of the nonlocal modes in Figs. 2 and 4 suggest that in this case the surface-plasmon peaks could be strongly blueshifted.

\section{ELECTRON ENERGY LOSS IN A WIRE}

We study now the energy losses due to plasmon excitation experienced by an electron traveling with velocity $v$ in the proximity of a metallic wire characterized by a nonlocal response function $\varepsilon(\mathbf{k}, \omega)$, as depicted in Fig. 1(a). The probability of losing energy $\hbar \omega$ per unit path length $\Gamma(\omega)$ is given by the action of the induced field $V^{\text {ind }}(\mathbf{r}, t)$ produced by the electron itself evaluated along the particle trajectory ( $\rho$ $\left.=\rho_{0}, \varphi=\varphi_{0}, z=v t\right)$ :

$$
-\left[\frac{\partial V^{\text {ind }}(\mathbf{r}, t)}{\partial z}\right]_{\text {traj }}=\int_{0}^{\infty} d \omega \omega \Gamma(\omega),
$$

where $V^{\text {ind }}(\mathbf{r}, t)$ is calculated from the $\omega, k$ component of the induced nonlocal potential driven by the traveling electron, obtained from Eqs. (A1) and (A17) in Appendix A. We identify terms at both sides of equality (8) and obtain the expression for the energy-loss probability per unit path length:

$$
\Gamma(\omega)=\frac{1}{2 \pi^{2} v} \operatorname{Im}\left[V^{\mathrm{ind}}\left(\rho_{0}, q_{z}=\omega v^{-1}, \omega\right)\right],
$$

with $\operatorname{Im}[x]$ as the imaginary part of $x$ and $V^{\text {ind }}\left(\rho_{0}, q_{z}\right.$ $\left.=\omega v^{-1}, \omega\right)$ as the Fourier component of the induced potential evaluated at $q_{z}=\omega v^{-1}$. Thus, for an electron traveling outside a metallic wire $\left(\rho_{0}>a\right)$, the probability of losing energy $\hbar \omega$ per unit length in this case is 

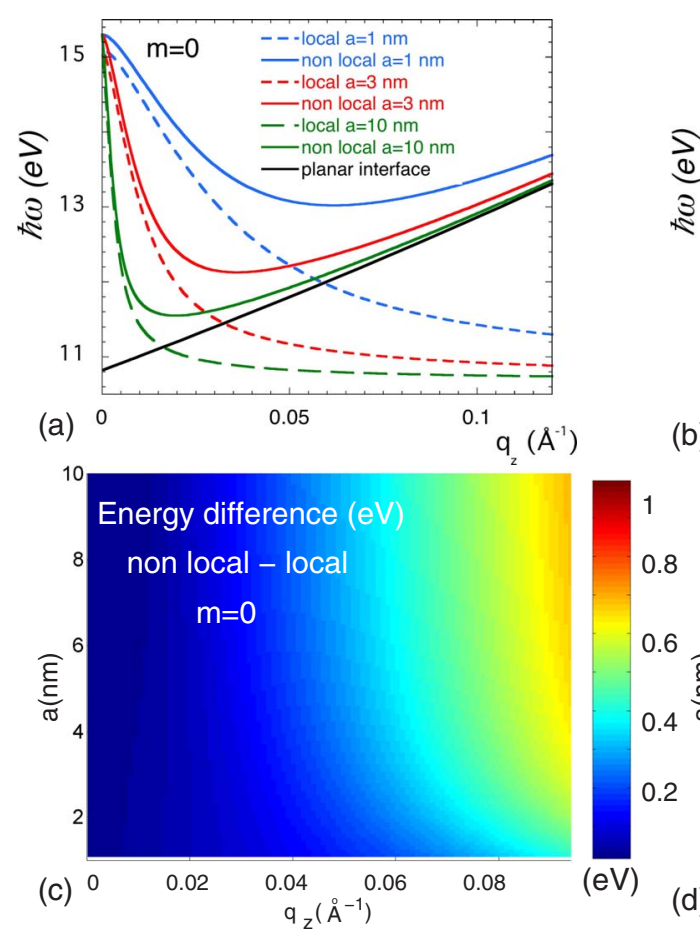

(b)
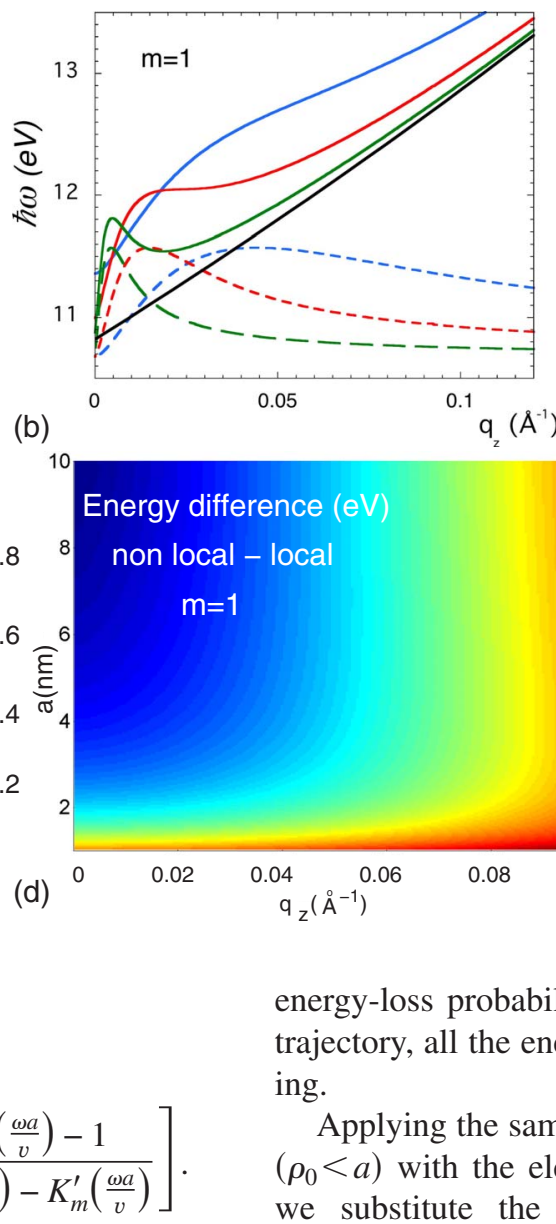
$[\varepsilon(k, \omega)=\varepsilon(\omega)]$, the local limit for the expression of the
If we neglect the $k$ dependence in the dielectric function

$$
\begin{aligned}
\Gamma(\omega)^{\left(\rho_{0}>a\right)}= & \frac{2}{\pi v^{2}} \sum_{m=-\infty}^{+\infty} K_{m}^{2}\left(\frac{\omega \rho_{0}}{v}\right) I_{m}^{\prime}\left(\frac{\omega a}{v}\right) \\
& \times \operatorname{Im}\left[\frac{\widetilde{\varepsilon}_{m}\left(a, \frac{\omega}{v}, \omega\right) I_{m}\left(\frac{\omega a}{v}\right) K_{m}\left(\frac{\omega a}{v}\right)-1}{\widetilde{\varepsilon}_{m}\left(a, \frac{\omega}{v}, \omega\right) K_{m}^{2}\left(\frac{\omega a}{v}\right) I_{m}^{\prime}\left(\frac{\omega a}{v}\right)-K_{m}^{\prime}\left(\frac{\omega a}{v}\right)}\right] .
\end{aligned}
$$$$
\text { intc }
$$

FIG. 4. (Color online) Plasmon mode energy (in $\mathrm{eV}$ ) corresponding to cylindrical cavities of radii $a=1 \mathrm{~nm}$ (blue lines), $a$ $=3 \mathrm{~nm}$ (red lines), and $a$ $=10 \mathrm{~nm}$ (green lines) for azimuthal numbers (a) $m=0$ and (b) $m=1$ as a function of parallel momentum component $q_{z}$. Same values as in Fig. 2 are used to characterize the nonlocal dielectric function of the metallic surrounding medium. [(c) and (d)] Contour plots showing the energy difference (in $\mathrm{eV}$ ) between the nonlocal and local approaches for $m=0$ and $m=1$, respectively. A blueshift is observed in all cases. energy-loss probability is recovered. ${ }^{16}$ For such an external trajectory, all the energy loss comes from the surface scattering.

Applying the same algebra, for trajectories inside the wire $\left(\rho_{0}<a\right)$ with the electron moving parallel to the wire axis, we substitute the expression of the nonlocal potential $V^{\text {ind }}(\mathbf{r}, t)$ inside the wire obtained from Eqs. (A19) and (A20) into Eq. (9). We obtain the following energy-loss probability per unit length:

$$
\Gamma(\omega)^{\left(\rho_{0}<a\right)}=\frac{2}{\pi v^{2}} \int_{0}^{\infty} \frac{Q d Q}{Q^{2}+\left(\frac{\omega}{v}\right)^{2}} \operatorname{Im}\left[\frac{-1}{\varepsilon(\mathbf{k}, \omega)}\right]+\frac{2}{\pi v^{2}} \sum_{m=-\infty}^{\infty} K_{m}^{\prime}\left(\frac{\omega a}{v}\right) \operatorname{Im}\left[\frac{\widetilde{\varepsilon_{m}}\left(a, \frac{\omega}{v}, \omega\right)}{\widetilde{\varepsilon}_{m}^{2}\left(\rho_{0}, \frac{\omega}{v}, \omega\right)} \frac{\widetilde{\varepsilon}_{m}\left(\rho_{0}, \frac{\omega}{v}, \omega\right) I_{m}\left(\frac{\omega \rho_{o}}{v}\right) K_{m}\left(\frac{\omega a}{v}\right)-1}{\widetilde{\varepsilon}_{m}(a) K_{m}^{2}\left(\frac{\omega a}{v}\right) I_{m}^{\prime}\left(\frac{\omega a}{v}\right)-K_{m}^{\prime}\left(\frac{\omega a}{v}\right)}\right]
$$

The first term in Eq. (11) is given by the direct bulk term and corresponds to the losses produced by bulk plasmons in an infinite medium. Therefore this term does not depend on the geometry of the cylinder-probe system since it is the energy-loss probability experienced by the probe in a nonbounded medium. The second term in Eq. (11) contains the contribution to the losses from the excitation of surface modes, plus a correction to the bulk term, arising from the presence of the boundaries (begrenzung). By making use of Eq. (4), it is straightforward to see that the surface contribution in both Eqs. (10) and (11) reduce to the expressions obtained with the use of a local dielectric function.
It should be noted that, in contrast to the results based on a local theory, in the limit of trajectories close to the interface $\left(\rho_{0} \rightarrow a\right)$, expression (10), outside the wire, and expression (11), inside the wire, lead to a finite value which varies continuously as the electron probe scans through the interface. In the local theory, the divergence of the surface contribution to the loss probability when $\left(\rho_{0} \rightarrow a\right)$ is a consequence of the unphysically large induced charge density on the interface, which leads to an image potential which diverges logarithmically with the impact parameter relative to the surface. In the case of a cylindrical target, this divergence is expressed through the lack of convergence of the sum of the 
contributions of the $m$ th multipolar terms. ${ }^{16}$ The use of a nonlocal dielectric function implies that only the first $m$ modes contribute effectively to the sum in Eqs. (10) and (11). This is a consequence of the smaller localization of the image charge in the nonlocal approach. Mathematically, the different contribution of the modes emerges as a consequence that the modified Bessel functions $J_{m}(Q a)$ involved in the definition of $\widetilde{\varepsilon}_{m}\left(\rho, q_{z}, \omega\right)$ in Eq. (3) take significant values only when $Q>m / a$. The contribution of large $m$ th terms to the energy loss naturally vanishes in Eqs. (10) and (11) because $\widetilde{\varepsilon}_{m}\left(a, q_{z}, \omega\right)$ becomes real, therefore not contributing to the losses. The continuity of the loss probability at the interface is a general feature of the dielectric formalism, and it occurs because of the continuity of the induced potential. This continuity is also present in the case of a local approach, when one needs to consider a momentum cutoff to prevent the divergence arising from large momentum contributions. This statement can be proven in the case of planar interfaces. In the general case of nonplanar targets, different cut-off procedures are used to evaluate the bulk and surface contributions. For the bulk term, a cut-off value $q_{c}$ is commonly used to mimic the effect of a circular collecting semiangle aperture $\theta_{c}=q_{c} / v$, whereas the surface term is regularized by truncating the multipolar series at a given term. The intrinsic differences in both procedures lead to a noncontinuous energy-loss probability at the interface. As mentioned above, in the case of a nonlocal approach, the momentum dependence of the dielectric function naturally guarantees that the contribution of large momentum values is discarded. Therefore no cutoff is needed.

We apply now expressions (10) and (11) to calculate the energy-loss spectra corresponding to a $100 \mathrm{keV}$ electron traveling along an aluminum cylinder of radius $a=3 \mathrm{~nm}$ characterized by a plasma energy $\hbar \omega_{p}=15.3 \mathrm{eV}$. In Fig. 5 we present the loss spectra in the range of energies of the planar surface and bulk plasmons. External (a) and internal (b) trajectories are considered at $0.1 \mathrm{~nm}$ from the surface $\left(\rho_{0}=3.1 \mathrm{~nm}\right.$ and $\rho_{0}=2.9 \mathrm{~nm}$, respectively). In Fig. 5(a) we show the loss spectra for an external trajectory, where the losses are produced by the excitation of wire surface plasmons. The spectrum consists of an energy-loss peak at $\approx 11 \mathrm{eV}$. We compare the spectra obtained within a local description of the dielectric response of the wire (red dashed line) and the nonlocal description (blue solid line). The nonlocal peak is shifted $0.3 \mathrm{eV}$ up in energy (blueshifted) with respect to the local result, with a broader distribution. The slight blueshift is consistent with the change in the dispersion relation of the modes in a metallic wire, shown previously in Fig. 2. The intensity of the surface-plasmon peak is lower in the nonlocal case due to the reduction in the momentum values which effectively contribute to the plasmon excitation. In the case of trajectories inside the wire $\left(\rho_{0}<a\right)$ through the metallic material, the energy-loss spectrum consists of the direct bulk contribution [first term in Eq. (11)] and a surface term [second term in Eq. (11)]. In Fig. 5(b) we show the surface terms (nonlocal as blue dotted-dashed line and local as red dashed line), as well as the sum of both terms in the nonlocal approach (black solid line). The resulting spectrum is similar to that in Fig. 5(a), and it consists of a surface peak at around $11 \mathrm{eV}$. The bulk-plasmon peak,
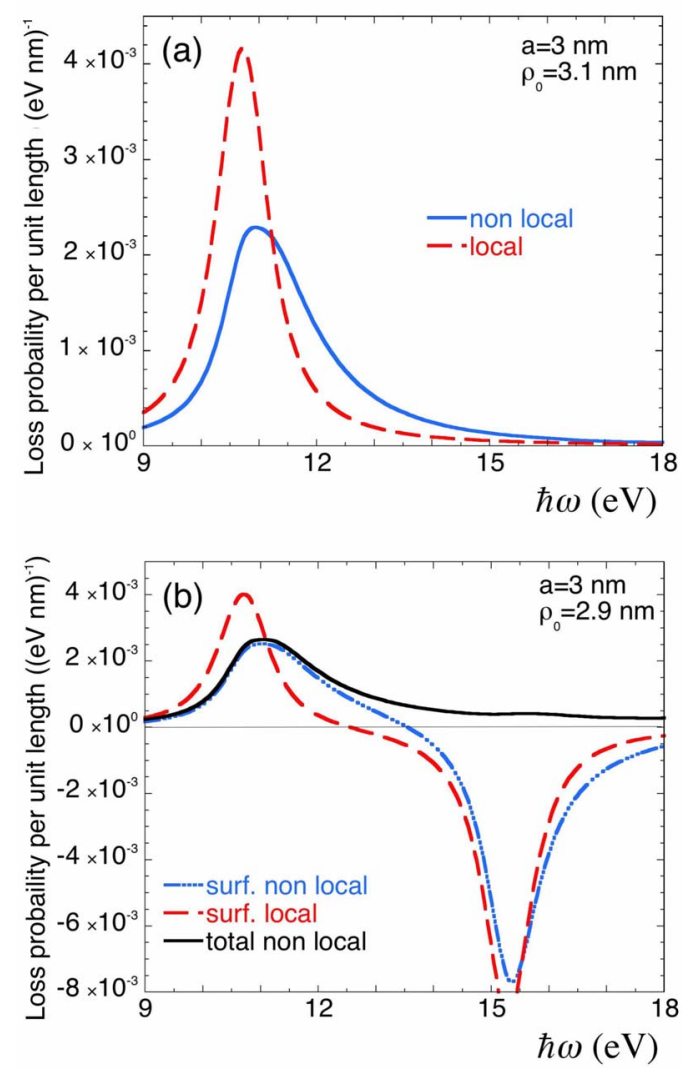

FIG. 5. (Color online) Energy-loss probability per unit length experienced by a $100 \mathrm{keV}$ electron traveling parallel to the axis of a cylinder with radius of $3 \mathrm{~nm}$. (a) corresponds to trajectories outside the wire, $\rho_{0}=3.1 \mathrm{~nm}$. Nonlocal (blue solid line) and local (red dashed line) results are displayed. (b) Total energy-loss probability per unit length (black solid line) corresponding to an internal trajectory, $\rho_{0}=2.9 \mathrm{~nm}$. Surface contributions are also shown as red line (local) and blue line (nonlocal). The parameters of the dielectric response function are $\omega_{p}=15.3 \mathrm{eV}$ and $\gamma=1 \mathrm{eV}$.

expected at $\omega_{p}$, is canceled almost exactly for this trajectory near the interface by the surface contribution, which becomes negative around $\omega_{p}$. This bulk-loss correcting effect by the induced potential is referred to as begrenzung and has been widely studied within the local approach. ${ }^{1,16,34,35,39,41,47}$ We can observe that the begrenzung effect is directly associated with the continuity of the energy-loss probability along the interface, which requires that the bulk peak vanishes inside the medium as the probe gets closer to the interface.

In Fig. 6 we analyze the dependence of the spectrum on the impact parameter $\rho_{0}$ for the same wire as in Fig. 5. For axial trajectories, due to the azimuthal symmetry of the surface charge density induced, only the $m=0$ mode contributes to the surface losses. The mode distribution in Fig. 3(a) indicates that for such a small wire, the energy of the $m=0$ mode lies approximately at $1 \mathrm{eV}$, a range not shown in Fig. 6(a). Therefore the loss spectrum consists exclusively of a peak centered at the bulk-plasmon energy for this central trajectory. Note that the probability of exciting bulk plasmons decays very fast around the interface. For penetrating trajectories, a few bumpy peaks above the bulk-plasmon peak can be observed. The origin of these peaks is connected 

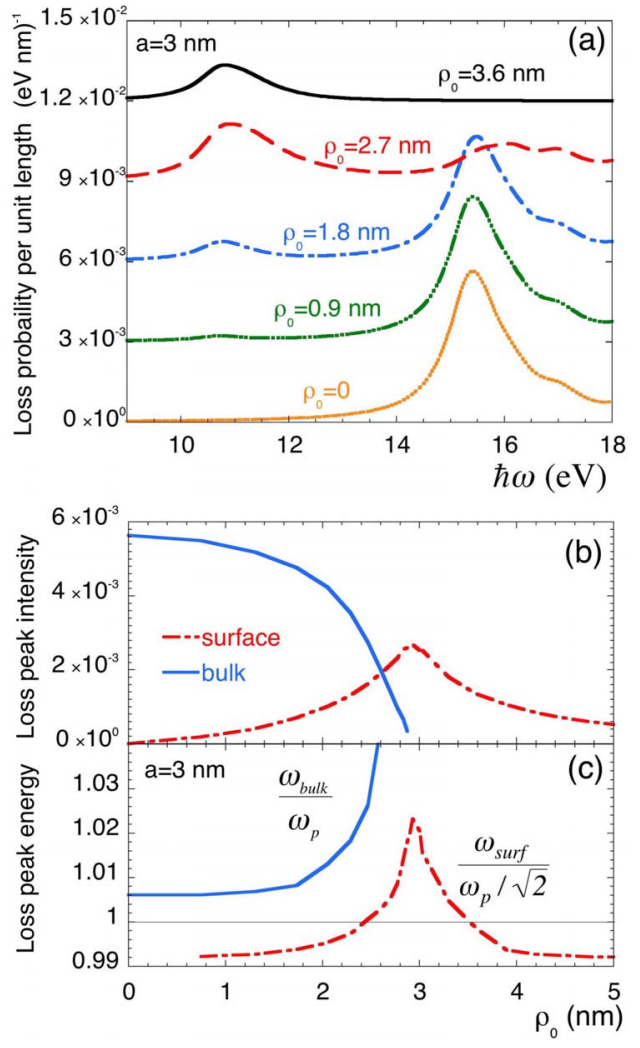

FIG. 6. (Color online) (a) Series of energy-loss spectra for different impact parameters $\rho_{0}$ ranging from $\rho_{0}=0$ (orange dotted line) to $\rho_{0}=3.6 \mathrm{~nm}$ (black solid line). The wire is the same as in Fig. 5 $(a=3 \mathrm{~nm})$. The spectra have been vertically shifted to improve clarity. (b) Impact parameter dependence of the intensity of each peak. The surface-loss peak is displayed as a red dotted-dashed line and the bulk-loss peak is displayed as a blue solid line. (c) Dispersion of both bulk (blue solid line) and surface (dotted-dashed line) peak energies as a function of the impact parameter. The energies of the peaks have been scaled to the bulk-plasmon frequency $\left(\omega_{p}\right)$ and planar surface-plasmon frequency $\left(\omega_{s}=\omega_{p} / \sqrt{2}\right)$, respectively.

with the discretization of the transverse $Q$ vector of the excitations imposed by the boundaries of the cylindrical cavity. The dispersion of the bulk plasmon $\left[\omega^{2}(k) \approx \omega_{p}^{2}+\beta^{2} k^{2}\right]$ together with this discretization (which selects particular $Q$ vectors) produces peaks at a set of energies above the bulk plasmon. These peaks are part of the begrenzung correction and tend to zero in the limit of a trajectory along the interface. The intensity and energy position of both surface and bulk losses can be traced in Figs. 6(b) and 6(c). As mentioned above, as the impact parameter increases when getting closer to the interface, modes with $m \geq 1$ are increasingly excited at energies close to the planar surface-plasmon energy $\hbar \omega_{s}=\hbar \omega_{p} / \sqrt{2} \approx 10.8 \mathrm{eV}$. Simultaneously and consistently with sum rules, the begrenzung correction also increases for trajectories closer to the interface [red dashed line in Fig. 6(a)], making smaller the intensity of the bulkplasmon peak [blue solid line in Fig. 6(b)]. At the interface and outside the wire, the bulk-plasmon peak vanishes, while the surface peak presents a maximum at the interface trajectory $\rho_{o}=a$ [red dashed line in Fig. 6(b)]. The energies of both peaks present a blueshift as the beam comes close to the
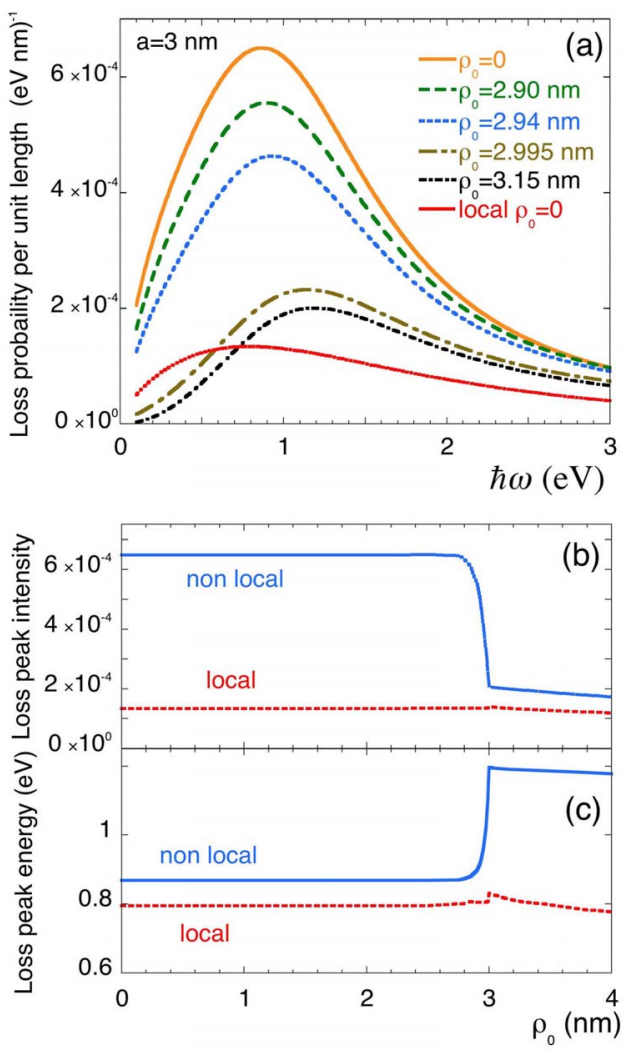

FIG. 7. (Color online) (a) Series of energy-loss spectra in a wire of radius $a=3 \mathrm{~nm}$ for different impact parameters $\rho_{0}$ ranging from $\rho_{0}=0$ (orange solid line) to $\rho_{o}=3.15 \mathrm{~nm}$ (black dotted-dashed line). The wire has the same dimensions as in Fig. $5(a=3 \mathrm{~nm})$. The local result for a central trajectory $\rho_{0}=0$ is also shown as a red solid line. (b) Impact parameter dependence of the intensity of the loss peaks shown in (a). Nonlocal (blue line) and local (red line) approaches are displayed. (c) Spectral shift of loss peaks presented in (a). The nonlocal case (blue line) presents an abrupt change in both intensity and spectral position compared to the local approach (red line).

interface $\left[\rho_{0} \rightarrow a\right.$ in Fig. 6(c)]. This shift is a consequence of a larger localization of the induced charge density in the proximity of the probe, which requires an increasingly larger number of $m$ th terms to correctly describe the induced potential, therefore increasing the energies of both bulk and surface modes.

It has been proven in the local approach that for very thin wires or for axial trajectories, the main contribution to the loss spectra comes from the $m=0$ term. ${ }^{49}$ The dispersion shown in Fig. 2(a) suggests that for a thin wire of radius $a$ $\left(a \ll v \omega^{-1}\right)$, the energy of the $m=0$ mode can be much smaller than the energy of the planar surface plasmon $\left(\hbar \omega_{s}\right.$ $\left.=\hbar \omega_{p} / \sqrt{2}\right)$. This is shown in Fig. 7(a), where we plot a series of very low-energy-loss spectra for different scanning impact parameters $\rho_{0}$. We observe that the shape of the loss peak in this region hardly changes when the probe is completely inside the wire (orange, green, and blue lines). The intensity of this peak is smaller than those found around $\omega_{p}$ in Fig. 6 but is still significant and therefore measurable. However, the intensity of this low-energy peak decreases very fast when the probe position is close to the interface. In 
Fig. 7(b) we present the dependence of the peak intensity on the impact parameter, with the energy-loss spectrum as a continuous function of the impact parameter $\rho_{0}$, This loss function presents a sudden change as the trajectory gets closer to the interface, $\rho_{0} \sim a$ [brown and black lines in Fig. 7(a)]. Also, the energy of this mode, shown in Fig. 7(c), features a similar change around the interface position. Two aspects of these results are noticeable. On one hand, the nonlocal peak [orange line in Fig. 7(a)] is significantly more intense than the local one (red line), a result that apparently contradicts the reduction in momentum space contributing to the plasmon excitation. On the other hand, the abrupt change in the peak intensity profile in Fig. 7(b) does not follow the smooth impact parameter dependence of typical surface excitations. This latter result has been experimentally tested in targets of different shapes but with larger dimensions than the targets considered here $(>10 \mathrm{~nm}) .{ }^{13}$ The abrupt change in the energy-loss peak shown in Fig. 7(c) rather resembles the features of bulk plasmons near an interface, suggesting the bulk nature of such one-dimensional (1D) plasmons in thin nanowires.

The dispersion in the dielectric response implies that the induced charge density is spread in a region of few atomic units near the interface. Therefore for very thin wires, the $m=0$ mode would consist of coherent oscillations of the electron gas in almost the full section of the wire, i.e., an excitation somehow similar to a bulk plasmon in onedimensional system. The connection of this anomalous effect with the 1D character of the wire is studied in Fig. 8. In the top graph [Fig. 8(a)], we present the energy-loss spectrum in the low-energy range for wires of two different sizes $(a$ $=1.5 \mathrm{~nm}$ and $a=10 \mathrm{~nm}$ ) in the case of axial trajectories $\left(\rho_{0}=0\right)$. We observe that the differences in the intensity between the local (red lines) and nonlocal (blue lines) approaches become relevant only for small radii. For radius larger than $10 \mathrm{~nm}$, the effect of dispersion is negligible. In Fig. 8(b), we show the intensity of this low-energy mode as a function of the reduced impact parameter $\rho_{0} / a$ for the same wires studied in (a). For a 20-nm-thick $(a=10 \mathrm{~nm})$ wire (dashed lines), the anomaly on the peak height at each side of the interface is not present, with both approaches (local in red and nonlocal in blue) giving similar values. However, for thin wires (solid lines), we observe an abrupt change in the intensity of the loss peak at the interface in the nonlocal approach, similar to the results presented for a wire with radius $a=3 \mathrm{~nm}$ in Fig. 7(b).

As pointed out previously in the discussion of the modes for very thin wires, we must note that the use of an isotropic dielectric function for such thin wires fails to reproduce the response of the electron bands in the transversal direction of the wire. However, this aspect would be relevant for extremely thin wires (1D electron gas) and would affect the induced charge density only in the wire cross section and not in the propagation direction along the wire axis, where the continuum of states assumed by the dielectric function is senseful. Even if the use of a more sophisticated description of the wire response in the transversal direction could shift the final energy of the modes, the results presented here showing a considerable blueshift of the plasmon response in such 1D structures remain valid and could be tested with
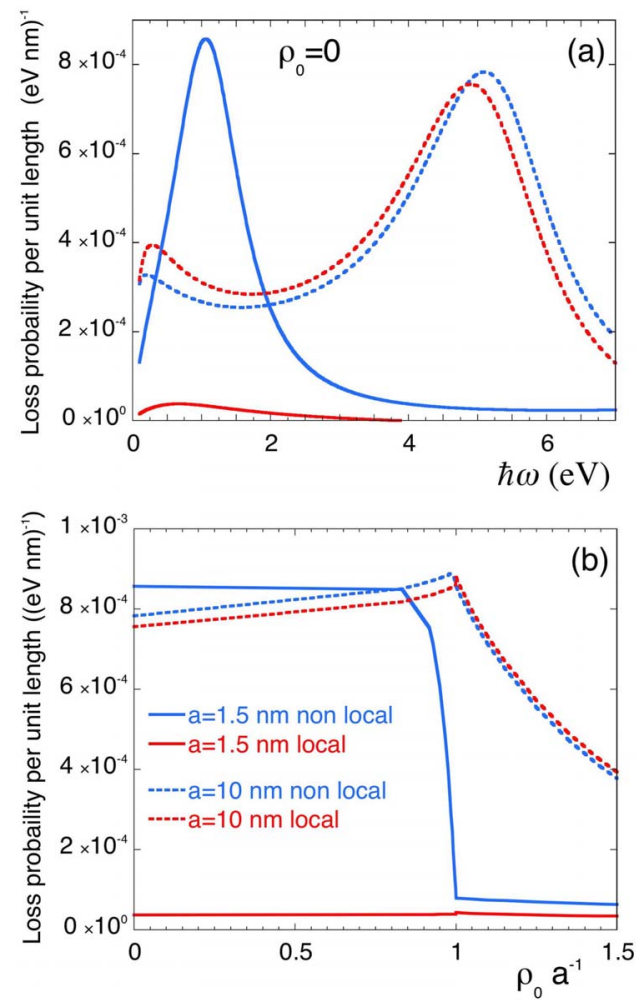

FIG. 8. (Color online) Energy-loss spectra for wires with radii $a=1.5 \mathrm{~nm}$ (solid lines) and $a=10 \mathrm{~nm}$ (dashed lines) in the range of $0-7 \mathrm{eV}$. The energy of the electron beam is $100 \mathrm{keV}$. (a) Spectra for axial trajectories of the electron $\left(\rho_{0}=0\right)$. Results with local (red lines) and nonlocal (blue lines) descriptions of the dielectric response are compared. Larger differences arise for small wires. (b) Impact parameter dependence of the energy-loss intensity corresponding to the peaks presented in (a). The labeling scheme stands for both (a) and (b). The large wire with a loss peak at around $5 \mathrm{eV}$ shows similar dependency for the local and the nonlocal descriptions, whereas the thin wire with the loss peak at around $1 \mathrm{eV}$ shows strong differences. An abrupt change in intensity is observed at the interface.

EELS experiments in monoatomic metallic chains. ${ }^{19}$

\section{ELECTRON ENERGY LOSS IN A CAVITY}

As pointed out in Sec. I, an electron beam has the ability to drill cylindrical cavities when going through a material. As the cylindrical hole surrounding the passing electron can be of very narrow dimension, we can expect that nonlocal effects might have a big impact in the characteristics of the surface plasmons excited by the electron beam in a nanocavity. Let us assume an electron moving near a cylindrical cavity of radius $a$ drilled in a metallic medium of dielectric function $\varepsilon(\mathbf{k}, \omega)$. We will assume that the electron travels parallel to the $Z$ axis of the hole, with velocity $v$ and impact parameter relative to the center of the cavity $\rho_{0}$, as depicted in Fig. 1(b).

Proceeding in the same way as in Sec. III and with use of the expressions for the nonlocal potential derived from Eqs. (B1) and (B2) in Appendix B, we obtain the following ex- 
pression for the energy-loss probability per unit length $\Gamma(\omega)$ for the case of the probe traveling through the cavity $\left(\rho_{0}\right.$ $<a)$ :

$$
\begin{aligned}
\Gamma(\omega)^{\left(\rho_{0}<a\right)}= & \frac{2}{\pi v^{2}} \sum_{m=-\infty}^{+\infty} I_{m}^{2}\left(\frac{\omega \rho_{0}}{v}\right) K_{m}^{\prime}\left(\frac{\omega a}{v}\right) \\
& \times \operatorname{Im}\left[\frac{\widetilde{\varepsilon}_{m}\left(a, \frac{\omega}{v}, \omega\right) I_{m}\left(\frac{\omega a}{v}\right) K_{m}\left(\frac{\omega a}{v}\right)-1}{\widetilde{\varepsilon}_{m}\left(a, \frac{\omega}{v}, \omega\right) I_{m}^{2}\left(\frac{\omega a}{v}\right) K_{m}^{\prime}\left(\frac{\omega a}{v}\right)-I_{m}^{\prime}\left(\frac{\omega a}{v}\right)}\right] .
\end{aligned}
$$

The function $\widetilde{\varepsilon}_{m}\left(\rho, q_{z}, \omega\right)$ for a particular radial coordinate $\rho$, parallel momentum $q_{z}$, and energy $\hbar \omega$ has been defined in Eq. (3). For trajectories inside the cavity, the only contribution to the losses arises from the surface excitations, given by the poles of expression (12).

In the case of electron trajectories through the surrounding metal, outside the cavity $\left(\rho_{0}>a\right)$, we use the expression for the nonlocal potential derived from Eq. (A19) with the coefficients from Eq. (B3) in Appendix B. In this case, we obtain the following the energy-loss probability per unit length:

$$
\Gamma(\omega)^{\left(\rho_{0}>a\right)}=\frac{2}{\pi v^{2}} \int_{0}^{\infty} \frac{Q d Q}{Q^{2}+q_{z}^{2}} \operatorname{Im}\left[\frac{-1}{\varepsilon(\mathbf{k}, \omega)}\right]+\frac{2}{\pi v^{2}} \sum_{m=-\infty}^{\infty} I_{m}^{\prime}\left(\frac{\omega a}{v}\right) \operatorname{Im}\left[\frac{\widetilde{\varepsilon}_{m}\left(a, \frac{\omega}{v}, \omega\right)}{\widetilde{\varepsilon}_{m}\left(\rho_{0}, \frac{\omega}{v}, \omega\right)^{2}} \frac{\widetilde{\varepsilon}_{m}\left(\rho_{0}, \frac{\omega}{v}, \omega\right) K_{m}\left(\frac{\omega \rho_{0}}{v}\right) I_{m}\left(\frac{\omega a}{v}\right)-1}{\left.\widetilde{\varepsilon}_{v}, \omega\right) I_{m}^{2}\left(\frac{\omega a}{v}\right) K_{m}^{\prime}\left(\frac{\omega a}{v}\right)-I_{m}^{\prime}\left(\frac{\omega a}{v}\right)}\right]
$$

Similar to that for the wire, the first term in Eq. (13) corresponds to the energy loss in a nonbounded medium (bulk losses), while the multipolar expansion contains the surface contribution and the correction to the bulk (begrenzung). If a local dielectric function is used, the energy-loss probabilities in Eqs. (12) and (13) recover the corresponding local expressions. ${ }^{16}$

In Fig. 9 we show the energy-loss spectra corresponding to a $100 \mathrm{keV}$ electron beam traveling near the interface of a cavity of radius $a=3 \mathrm{~nm}$ drilled in a metal characterized by a nonlocal dielectric response given by Eq. (1). Both the trajectories through the cavity [Fig. 9(a)] and through the metal outside the cavity [Fig. 9(b)] are considered. The spectra consist basically of two surface peaks, one at $11 \mathrm{eV}$, at slightly larger energy than the planar surface plasmon, and a second peak at $14.6 \mathrm{eV}$, near the energy of the bulk plasmon. The latter peak is originated from the surface contribution of the $m=0$ cavity mode. As expected from the cavity modes dispersion in Figs. 4(a) and 4(b) and considering the small $q_{z}$ value of this situation $\left(q_{z}=\omega v^{-1} \sim 0.01 \AA^{-1}\right)$, both local (red line) and nonlocal (blue line) $m=0$ modes $(\approx 14.6 \mathrm{eV})$ do not differ in the energy position. The peak around $11 \mathrm{eV}$ arises from the remaining $m \neq 0$ terms, and nonlocal effects are more relevant for these higher-order terms, as observed in the dispersion of modes of Figs. 4(b) and 4(d). Therefore a blueshift of the low-energy peak at around $11 \mathrm{eV}$ is produced with respect to the local approach (red dashed line) when nonlocal effects are considered (blue solid line). The reduction in the momentum values contributing to the plasmon excitation has also an effect in this difference. In the case of the electron trajectories outside the cavity, through the metal [Fig. 9(b)], we find both surface peaks at the same positions as in Fig. 9(a) and also the cancellation of the bulkplasmon peak by the begrenzung correction, with some modulation above the bulk-plasmon energy due to the dispersion and modulation of the nonlocal begrenzung, similar to those in Fig. 6(a). The spectra calculated for electron beams traveling at both sides of the interface in Fig. 9(a) (blue solid line) and Fig. 9(b) (black solid line) are very similar with the use of the nonlocal approach, this being a consequence of the natural continuity discussed in Sec. III.
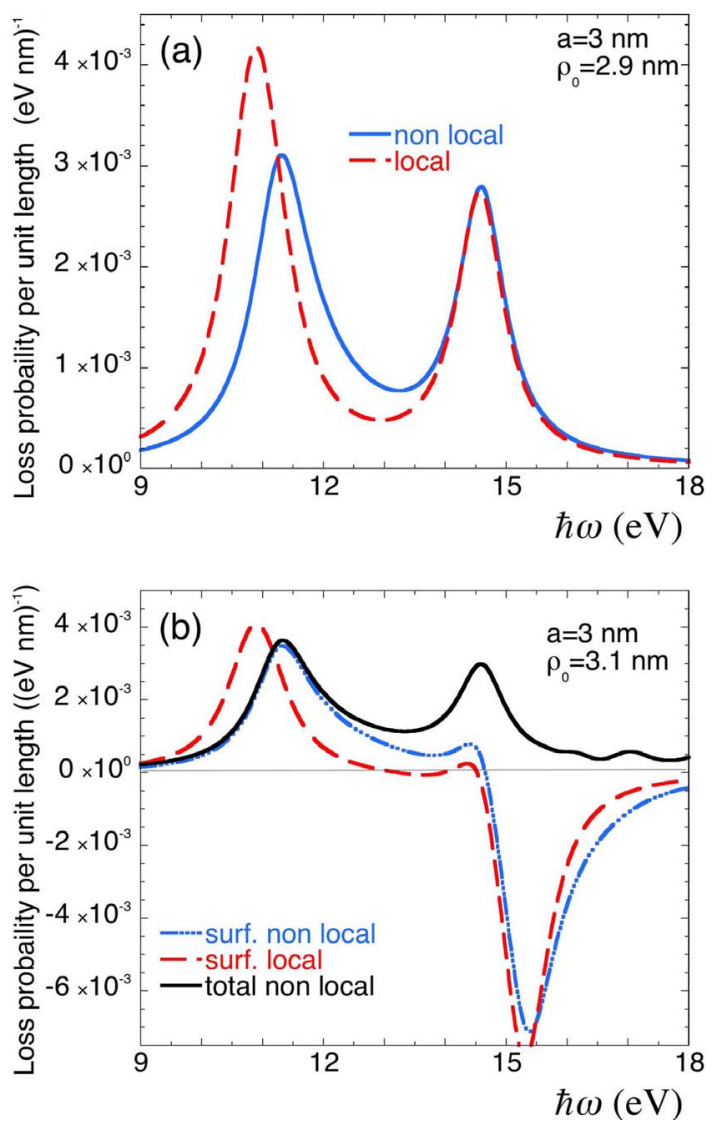

FIG. 9. (Color online) Energy-loss probability per unit length experienced by a $100 \mathrm{keV}$ electron traveling parallel to the axis of a cylindrical cavity with radius of $3 \mathrm{~nm}$. (a) corresponds to trajectories inside the cavity, $\rho_{0}=2.9 \mathrm{~nm}$, with the uses of local (red dashed line) and nonlocal (blue solid line) approaches. (b) corresponds to a trajectory outside the cavity, in the metal, $\rho_{0}=3.1 \mathrm{~nm}$. The totalenergy-loss probability per unit path length is plotted as a black solid line, whereas the surface contributions are displayed as red dashed lines (local) and blue dotted lines (nonlocal). 


\section{CONCLUSIONS}

We have developed a theoretical framework that allows the inclusion of nonlocal effects in the excitation of plasmons by fast electron beams. The specular reflection model has been used to characterize the momentum dependence of the metallic material with use of a plasmon pole approximation to characterize the dielectric response. This response accounts for the main features of nonlocality at the interfaces. Compared to a local approach, a blueshift in the energy of the plasmons is obtained, especially for very thin wires, where the nonlocal induced charge at the walls of wires and cavities interact more strongly. A very low-energy mode is found for very thin wires that resembles the behavior of a 1D plasmon, and it presents remarkable differences in the position and intensity of the excitation when nonlocal effects are consider. The excitation of surface and bulk plasmons are traced for different impact parameters, and a natural continuity of the spectrum of losses is found when the nonlocal approach is applied. Considering the progressive minimization of the size of wires in different technological approaches and the ability of electrons to drill thin holes in their trajectory, nonlocal effects should be considered and included in the optical response of these metallic systems for an accurate description of plasmons and optimal tuning of resonant situations.

\section{ACKNOWLEDGMENTS}

The authors would like to acknowledge comments by D. Sanchez Portal, J. M. Pitarke, and P. M. Echenique. Financial support from the Department of Industry of the Basque Government and from Gipuzkoako Foru Aldundia through the Etortek project NANOTRON is also acknowledged.

\section{APPENDIX A: NONLOCAL POTENTIAL IN A WIRE}

We derive in this appendix the expressions for the nonlocal potentials around a metallic nanowire with use of the SRM. ${ }^{57}$ Both external and internal trajectories are considered. First we assume an electron moving with velocity $v$ outside a cylindrical wire of radius $a$ parallel to the $Z$ axis at a radial distance $\rho_{0}$ from the center of the wire $\left(\rho_{0}>a\right)$, as shown in Fig. 1(a). We characterize the metal in the wire by a nonlocal dielectric function $\varepsilon(\mathbf{k}, \omega)$ taken from Eq. (1) within the plasmon pole approximation, but any other nonlocal dielectric function could be used with generality. Laplace equation can be separated in cylindrical coordinates $(\rho, \varphi, z)$, and Poisson's equation solved through an expansion of Green's functions in cylindrical coordinates. ${ }^{89}$ The potential $V(\mathbf{r})$ outside the wire (assumed to be the vacuum) is then given by

$$
\begin{aligned}
V(\mathbf{r}, \omega)= & V^{d}(\mathbf{r}, \omega)+V^{\text {ind }}(\mathbf{r}, \omega)=\frac{-1}{2 \pi} \int_{-\infty}^{\infty} d q_{z} e^{i q_{z} z} \\
& \times \sum_{m=-\infty}^{+\infty} 4 \pi \delta\left(\omega-q_{z} v\right) e^{i m \varphi}\left[I_{m}\left(q_{z} \rho_{0}\right) K_{m}\left(q_{z} \rho\right)\right. \\
& \left.\times \theta\left(\rho-\rho_{0}\right)+K_{m}\left(q_{z} \rho_{0}\right) I_{m}\left(q_{z} \rho\right) \theta\left(\rho_{0}-\rho\right)\right]
\end{aligned}
$$

$$
+\frac{1}{2 \pi} \int_{-\infty}^{\infty} d q_{z} e^{i q_{z} z} \sum_{m=-\infty}^{+\infty} A_{m} e^{i m \varphi} K_{m}\left(q_{z} \rho\right)
$$

where the first term $V^{d}(\mathbf{r}, \omega)$ is the potential produced directly by the moving charge and the second term $V^{\text {ind }}(\mathbf{r}, \omega)$ is the potential produced by the charge induced at the wire. $I_{m}(x)$ and $K_{m}(x)$ are the modified Bessel functions of order $m,{ }^{87}$ and $\theta(x)$ is the Heaviside function. Therefore, the problem reduces to finding the $A_{m}$ coefficients in the induced term of the potential.

Inside the wire $(\rho<a)$, we can write

$$
\begin{gathered}
\nabla \cdot \mathbf{D}=0, \\
\nabla \times \mathbf{E}=0 .
\end{gathered}
$$

Following Ritchie and Marusak, ${ }^{57}$ we will assume an infinite fictitious medium in order to solve for the fields inside the wire. This medium satisfies the following conditions: (i) Maxwell's equations are continued to the $\rho<a$ region of the infinite medium. (ii) The fields inside the real wire are the same as the ones of the infinite medium with the same response function. (iii) The normal component of the displacement $\mathbf{D}_{\rho}$ is discontinuous in $\rho=a$ in this infinite system, but the tangential components are continuous. Therefore, we introduce a uniform dielectric medium with a fictitious cylindrical surface of charge at $\rho=a$ which acts as a source for $D$. Because of this, $\nabla \cdot \mathbf{D}=0$ (which normally holds throughout an infinite, continuous medium) does not hold on the surface of the wire. We introduce now the potential function $V_{D}(\mathbf{r})$ defined by

$$
\mathbf{D}(\mathbf{r})=-\nabla V_{D}(\mathbf{r}) .
$$

We note that inside the cylindrical surface of the fictitious medium, $V(\mathbf{r})$ and $V_{D}(\mathbf{r})$ must be of the form

$$
V(\mathbf{r})=\frac{1}{2 \pi} \int_{-\infty}^{+\infty} d q_{z} e^{i q_{z} z} \sum_{m=-\infty}^{+\infty} e^{i m \varphi} \widetilde{V}_{m}\left(\rho, q_{z}\right),
$$

and

$$
V_{D}(\mathbf{r})=\frac{1}{2 \pi} \int_{-\infty}^{+\infty} d q_{z} e^{i q_{z} z} \sum_{m=-\infty}^{+\infty} e^{i m \varphi} \widetilde{V}_{D m}\left(\rho, q_{z}\right) .
$$

Equations (A2) and (A3) together with Eq. (A4) reduce to

$$
\nabla^{2} V_{D}=0 .
$$

Multiplying by $e^{-i \mathbf{k r}}$ and integrating on $\mathbf{r}$, we get

$$
\begin{aligned}
& -k^{2} V_{D}(\mathbf{k})+a \sum_{m=-\infty}^{+\infty} \frac{1}{2 \pi} C_{m} \\
& \times\left[\int_{-\infty}^{+\infty} d z \int_{0}^{2 \pi} d \varphi e^{-i \mathbf{k} r} \int_{-\infty}^{+\infty} d q_{z} e^{i q_{z} z} e^{i m \varphi}\right]_{\rho=a}=0,
\end{aligned}
$$




$$
C_{m}=-\left[\frac{d \tilde{V}_{D m}}{d \rho}\right]_{\rho=a^{-}}+\left[\frac{d \tilde{V}_{D m}}{d \rho}\right]_{\rho=a^{+}} .
$$

We expand $e^{-i \mathbf{k} r}$ in cylindrical coordinates by using the relation $^{89}$

$$
e^{-i \mathbf{k} r}=e^{-i q_{z} z} \sum_{p=-\infty}^{+\infty}(-i)^{p} e^{i p(\theta-\varphi)} J_{p}(Q \rho),
$$

where $k^{2}=Q^{2}+q_{z}^{2}, \theta$ and $\varphi$ are the azimuthal angles of $\mathbf{k}$ and $\mathbf{r}$, respectively, and $J_{p}(Q \rho)$ is the Bessel function of order $p$. After integrating Eq. (A9) over $z$ and $\varphi$, we get

$$
V_{D}(\mathbf{k}, \omega)=\sum_{m=-\infty}^{+\infty} 2 \pi a C_{m}(-i)^{m} e^{i m \theta} \frac{J_{m}(Q a)}{Q^{2}+q_{z}^{2}},
$$

where

$$
V(\mathbf{k}, \omega)=\sum_{m=-\infty}^{+\infty} 2 \pi a C_{m}(-i)^{m} e^{i m \theta} \frac{J_{m}(Q a)}{\left(Q^{2}+q_{z}^{2}\right) \varepsilon(\mathbf{k}, \omega)} .
$$

We come back to the space coordinate using the following scheme for the Fourier transform:

$$
\begin{gathered}
f(\mathbf{k}, \omega)=\int_{-\infty}^{+\infty} d \mathbf{r} \int_{-\infty}^{+\infty} d t e^{-i(\mathbf{k} \cdot \mathbf{r}-\omega t)} f(\mathbf{r}, t), \\
f(\mathbf{r}, t)=\frac{1}{(2 \pi)^{4}} \int_{-\infty}^{+\infty} d \mathbf{k} \int_{-\infty}^{+\infty} d \omega e^{i(\mathbf{k} \cdot \mathbf{r}-\omega t)} f(\mathbf{k}, \omega) .
\end{gathered}
$$

We obtain

$$
\begin{aligned}
V_{D}(\mathbf{r}, \omega)= & \frac{a}{(2 \pi)^{2}} \sum_{m=-\infty}^{+\infty} C_{m} e^{i m \varphi} \\
& \times \int_{-\infty}^{+\infty} d q_{z} e^{i q_{z} z} \int_{0}^{+\infty} Q d Q \frac{J_{m}(Q a) J_{m}(Q \rho)}{Q^{2}+q_{z}^{2}}
\end{aligned}
$$

and

$$
\begin{aligned}
V(\mathbf{r}, \omega)= & \frac{a}{(2 \pi)^{2}} \sum_{m=-\infty}^{+\infty} C_{m} e^{i m \varphi} \\
& \times \int_{-\infty}^{+\infty} d q_{z} e^{i q_{z} z} \int_{0}^{+\infty} Q d Q \frac{J_{m}(Q a) J_{m}(Q \rho)}{\left(Q^{2}+q_{z}^{2}\right) \varepsilon(\mathbf{k}, \omega)} .
\end{aligned}
$$

Now we will take this potential as the one corresponding to our real system inside the wire $(\rho<a)$ and apply the boundary conditions at $\rho=a$ with the potential given by Eq. (A1), which is valid outside the wire $(\rho>a) . D_{\rho}(\mathbf{r})$ is obtained in both cases as the derivative of the potential $V_{D}(\mathbf{r})$, as $\mathbf{D}(\mathbf{r})=-\nabla V_{D}(\mathbf{r})$, and has to be also continuous at $\rho=a$. We solve equations of continuity for $V\left(a^{+}\right)=V\left(a^{-}\right)$and normal component of $D_{\rho}\left(a^{+}\right)=D_{\rho}\left(a^{-}\right)$and obtain for $A_{m}$

$$
\begin{aligned}
A_{m}= & 4 \pi \delta\left(\omega-q_{z} v\right) K_{m}\left(q_{z} \rho_{0}\right) I_{m}^{\prime}\left(q_{z} a\right) \\
& \times\left[\frac{\widetilde{\varepsilon}_{m}\left(a, q_{z}, \omega\right) K_{m}\left(q_{z} a\right) I_{m}\left(q_{z} a\right)-1}{\widetilde{\varepsilon}_{m}\left(a, q_{z}, \omega\right) K_{m}^{2}\left(q_{z} a\right) I_{m}^{\prime}\left(q_{z} a\right)-K_{m}^{\prime}\left(q_{z} a\right)}\right],
\end{aligned}
$$

with

$$
\frac{1}{\widetilde{\varepsilon}_{m}\left(a, q_{z}, \omega\right)}=\int_{0}^{\infty} \frac{J_{m}^{2}(Q a)}{\left(Q^{2}+q_{z}^{2}\right) \varepsilon(\mathbf{k}, \omega)} Q d Q .
$$

Introducing this coefficient $A_{m}$ into Eq. (A1), we obtain the expression of the nonlocal potential outside the wire. The modes of the wire system are given by the zeros of the denominator of this function, as explicitly written in Eq. (2).

For a case where the electron is traveling parallel to the wire axis, inside the wire, i.e., $\left(\rho_{0}<a\right)$, the potential of interest is now that inside the wire. Analogous to the case of the external trajectory, we also apply the SRM, introducing a cylindrical surface charge density at the wire surface. This allows us to express the potential inside the wire as

$$
\begin{aligned}
V(\mathbf{r}, \omega)= & V^{d}(\mathbf{r}, \omega)+V^{\mathrm{ind}}(\mathbf{r}, \omega) \\
= & \frac{-1}{2 \pi} \int_{-\infty}^{\infty} d q_{z} e^{i q_{z} z} \sum_{m=-\infty}^{+\infty} 4 \pi \delta\left(\omega-q_{z} v\right) e^{i m \varphi} \\
& \times \int_{0}^{\infty} d Q Q \frac{J_{m}(Q \rho) J_{m}\left(Q \rho_{0}\right)}{\left(Q^{2}+q_{z}^{2}\right) \varepsilon(\mathbf{k}, \omega)} \\
& +\frac{1}{(2 \pi)} \int_{-\infty}^{\infty} d q_{z} e^{i q_{z} z} \sum_{m=-\infty}^{+\infty} B_{m} e^{i m \varphi} \int_{0}^{\infty} d Q Q \\
& \times \frac{J_{m}(Q a) J_{m}(Q \rho)}{\left(Q^{2}+q_{z}^{2}\right) \varepsilon(\mathbf{k}, \omega)} .
\end{aligned}
$$

The first term in Eq. (A19) is the direct Coulomb term corresponding to the potential from the infinite bulk medium, and the second term corresponds to the induced potential due to the surface modes plus the correction to the bulk modes due to the presence of the surface. After a similar algebraic treatment as in the previous case, we obtain for a trajectory inside the wire $\left(\rho_{0}<a\right)$ the following expression for the coefficient $B_{m}^{\left(\rho_{0}<a\right)}$, giving the surface contribution:

$$
\begin{aligned}
B_{m}^{\left(\rho_{0}<a\right)}= & 4 \pi \delta\left(\omega-q_{z} v\right) K_{m}^{\prime}\left(q_{z} a\right) \frac{\widetilde{\varepsilon}_{m}\left(a, q_{z}, \omega\right)}{\widetilde{\varepsilon}_{m}\left(\rho_{0}, q_{z}, \omega\right)} \\
& \times\left[\frac{\widetilde{\varepsilon}_{m}\left(\rho_{0}, q_{z}, \omega\right) I_{m}\left(q_{z} \rho_{0}\right) K_{m}\left(q_{z} a\right)-1}{\widetilde{\varepsilon}_{m}\left(a, q_{z}, \omega\right) K_{m}^{2}\left(q_{z} a\right) I_{m}^{\prime}\left(q_{z} a\right)-K_{m}^{\prime}\left(q_{z} a\right)}\right] .
\end{aligned}
$$

The zeros of the denominator coincide, as it should be, with those obtained for the external trajectory. An extra zero corresponding to the bulk mode $\widetilde{\varepsilon}_{m}\left(\rho_{0}, q_{z}, \omega\right)=0$ shows explicitly the correction to the bulk mode by the presence of the surface (begrenzung). 


\section{APPENDIX B: NONLOCAL POTENTIAL IN A CYLINDRICAL CAVITY}

We assume now an electron moving with velocity $v$ inside a cylindrical cavity of radius $a$ parallel to the $Z$ axis at a radial distance $\rho_{0}$ from the center of the cavity $\left(\rho_{0}<a\right)$, as shown in Fig. 1(b). We characterize the metal surrounding the cavity by a nonlocal dielectric function $\varepsilon(\mathbf{k}, \omega)$ taken from Eq. (1). We follow the same treatment separating Laplace equation in cylindrical coordinates and solving Poisson's equation. The potential $V(\mathbf{r})$ inside the cavity (assumed to be vacuum) is then given by

$$
\begin{aligned}
V(\mathbf{r}, \omega)= & V^{d}(\mathbf{r}, \omega)+V^{\text {ind }}(\mathbf{r}, \omega) \\
= & \frac{-1}{2 \pi} \int_{-\infty}^{\infty} d q_{z} e^{i q_{z} z} \sum_{m=-\infty}^{+\infty} 4 \pi \delta\left(\omega-q_{z} v\right) e^{i m \varphi} \\
& \times\left[I_{m}\left(q_{z} \rho_{0}\right) K_{m}\left(q_{z} \rho\right) \theta\left(\rho-\rho_{0}\right)\right. \\
& \left.+K_{m}\left(q_{z} \rho_{0}\right) I_{m}\left(q_{z} \rho\right) \theta\left(\rho_{0}-\rho\right)\right] \\
& +\frac{1}{2 \pi} \int_{-\infty}^{\infty} d q_{z} e^{i q_{z} z} \sum_{m=-\infty}^{+\infty} E_{m} e^{i m \varphi} I_{m}\left(q_{z} \rho\right),
\end{aligned}
$$

where the first term $V^{d}(\mathbf{r}, \omega)$ is the potential produced directly by the moving charge in an infinite medium and the second term $V^{\text {ind }}(\mathbf{r}, \omega)$ is the potential produced by the charge induced in the material outside the cavity. The generation of the nonlocal surface charge density at the cylindrical boundaries of the cavity follows strictly the same procedure as in Appendix A. The continuity of the potentials and the displacements at the boundaries lead with the use of the same algebra to the value of the coefficient:

$$
\begin{aligned}
E_{m}= & 4 \pi \delta\left(\omega-q_{z} v\right) I_{m}\left(q_{z} \rho_{0}\right) K_{m}^{\prime}\left(q_{z} a\right) \\
& \times\left[\frac{\widetilde{\varepsilon}_{m}\left(a, q_{z}, \omega\right) K_{m}\left(q_{z} a\right) I_{m}\left(q_{z} a\right)-1}{\widetilde{\varepsilon}_{m}\left(a, q_{z}, \omega\right) I_{m}^{2}\left(q_{z} a\right) K_{m}^{\prime}\left(q_{z} a\right)-I_{m}^{\prime}\left(q_{z} a\right)}\right],
\end{aligned}
$$

with the expression for $\widetilde{\varepsilon}_{m}\left(a, q_{z}, \omega\right)$ in Eq. (A18). The nonlocal modes for the cylindrical cavity are given by the zeros of the denominator of this coefficient, as explicitly written in Eq. (6).

For a trajectory through the material surrounding the cavity $\left(\rho_{0}>a\right)$, the expression for the nonlocal potential is exactly the same as in Eq. (A19), since this general form of potential corresponds to a bulk term, plus the excitation of a cylindrical surface scatterer. When the conditions of continuity are applied at the cylindrical cavity, the explicit expression for the surface coefficient $B_{m}^{\left(\rho_{0}>a\right)}$ can be obtained as follows:

$$
\begin{aligned}
B_{m}^{\left(\rho_{0}>a\right)}= & 4 \pi \delta\left(\omega-q_{z} v\right) I_{m}^{\prime}\left(q_{z} a\right) \frac{\widetilde{\varepsilon}_{m}\left(a, q_{z}, \omega\right)}{\widetilde{\varepsilon}_{m}\left(\rho_{0}, q_{z}, \omega\right)} \\
& \times\left[\frac{\widetilde{\varepsilon}_{m}\left(\rho_{0}, q_{z}, \omega\right) K_{m}\left(q_{z} \rho_{0}\right) I_{m}\left(q_{z} a\right)-1}{\widetilde{\varepsilon}_{m}\left(a, q_{z}, \omega\right) I_{m}^{2}\left(q_{z} a\right) K_{m}^{\prime}\left(q_{z} a\right)-I_{m}^{\prime}\left(q_{z} a\right)}\right] .
\end{aligned}
$$

Together with the surface modes, as it happens in a penetrating trajectory through the wire, in the current case of a trajectory through the material outside the cavity a correction to the bulk mode is also present as an extra zero in the denominator of the coefficient $B_{m}^{\left(\rho_{0}>a\right)}$. *aizpurua@ehu.es

${ }^{1}$ R. H. Ritchie, Phys. Rev. 106, 874 (1957).

${ }^{2}$ C. F. Bohren and D. R. Huffman, Absorption and Scattering of Light by Small Particles (Wiley, Berlin, 1998).

${ }^{3}$ C. J. Powell and J. B. Swan, Phys. Rev. 115, 869 (1959); 116, 81 (1959); 118, 640 (1959).

${ }^{4}$ J. Nelayah, M. Kociak, O. Stäphan, F. J. García de Abajo, M. Tencä, L. Henrard, D. Taverna, I. Pastoriza-Santos, L. M. LizMarzán, and C. Colliex, Nat. Phys. 3, 348 (2007).

${ }^{5}$ N. Yamamoto, K. Araya, and F. J. García de Abajo, Phys. Rev. B 64, 205419 (2001).

${ }^{6}$ J. T. van Wijngaarden, E. Verhagen, A. Polman, C. E. Ross, H. J. Lezec, and H. A. Atwater, Appl. Phys. Lett. 88, 221111 (2006).

${ }^{7}$ C. E. Hofmann, E. J. R. Vesseur, L. A. Sweatlock, H. J. Lezec, F. J. García de Abajo, A. Polman, and H. A. Atwater, Nano Lett. 7, 3612 (2007).

${ }^{8}$ M. V. Bashevoy, F. Jonsson, K. F. MacDonald, Y. Chen, and N. I. Zheludev, Opt. Express 15, 11313 (2007).

${ }^{9}$ P. E. Batson, N. Dellby, and O. L. Krivanek, Nature (London) 418, 617 (2002).

${ }^{10}$ P. E. Batson, Phys. Rev. Lett. 49, 936 (1982).

${ }^{11}$ M. Achèche, C. Colliex, H. Kohl, A. Nourtier, and P. Trebbia, Ultramicroscopy 20, 99 (1986).

${ }^{12}$ Z. L. Wang and J. M. Cowley, Ultramicroscopy 21, 77 (1987);
21, 335 (1987); 21, 347 (1987); 23, 97 (1987).

${ }^{13}$ D. Ugarte, C. Colliex, and P. Trebbia, Phys. Rev. B 45, 4332 (1992).

${ }^{14}$ F. Ouyang, P. E. Batson, and M. Isaacson, Phys. Rev. B 46, 15421 (1992)

${ }^{15}$ C. A. Walsh, Philos. Mag. A 59, 227 (1989).

${ }^{16}$ N. Zabala, A. Rivacoba, and P. M. Echenique, Surf. Sci. 209, 465 (1989).

${ }^{17}$ B. W. Reed, J. M. Chen, N. C. MacDonald, J. Silcox, and G. F. Bertsch, Phys. Rev. B 60, 5641 (1999).

${ }^{18}$ T. Hanrath and B. A. Korgel, Nano Lett. 4, 1455 (2004).

${ }^{19}$ T. Nagao, S. Yaginuma, T. Inaoka, and T. Sakurai, Phys. Rev. Lett. 97, 116802 (2006).

${ }^{20}$ J. Kikkawa, S. Takeda, Y. Sato, and M. Terauchi, Phys. Rev. B 75, 245317 (2007).

${ }^{21}$ Y. Saito, H. Shinohara, and A. Ohshita, Jpn. J. Appl. Phys., Part 2 30, L1068 (1991).

${ }^{22}$ A. Lucas, G. Gensterblum, J. J. Pireaux, P. A. Thiry, R. Caudano, J. P. Vigneron, P. Lambin, and W. Kratschmer, Phys. Rev. B 45, 13694 (1992).

${ }^{23}$ J. W. Keller and M. A. Coplan, Chem. Phys. Lett. 193, 89 (1992).

${ }^{24}$ R. Kuzuo, M. Terauchi, M. Tanaka, Y. Saito, and H. Shinohara, Phys. Rev. B 49, 5054 (1994). 
${ }^{25}$ R. Kuzuo, M. Terauchi, M. Tanaka, Y. Saito, and Y. Achiba, Phys. Rev. B 51, 11018 (1995).

${ }^{26}$ T. Stőckli, J. M. Bonard, A. Châtelain, Z. L. Wang, and P. Stadelmann, Phys. Rev. B 61, 5751 (2000).

${ }^{27}$ T. Pichler, M. Knupfer, M. S. Golden, J. Fink, and T. Cabioc'h, Phys. Rev. B 63, 155415 (2001).

${ }^{28}$ V. P. Dravid, X. Lin, Y. Wang, X. K. Wang, A. Yee, and R. P. H. C. J. B. Ketterson, Science 259, 1601 (1993).

${ }^{29}$ L. A. Bursill, P. A. Stadelmann, J. L. Peng, and S. Prawer, Phys. Rev. B 49, 2882 (1994).

${ }^{30}$ M. Kociak, L. Henrard, O. Stéphan, K. Suenaga, and C. Colliex, Phys. Rev. B 61, 13936 (2000).

${ }^{31}$ B. W. Reed and M. Sarikaya, Phys. Rev. B 64, 195404 (2001).

${ }^{32}$ P. M. Echenique and J. B. Pendry, J. Phys. C 8, 2936 (1975).

${ }^{33}$ F. Fujimoto and K. Komki, J. Phys. Soc. Jpn. 25, 1769 (1968).

${ }^{34}$ P. M. E. R. Nuñez and R. H. Ritchie, J. Phys. C 13, 4229 (1980).

${ }^{35}$ M. Schmeits, J. Phys. C 14, 1203 (1981).

${ }^{36}$ H. Kohl, Ultramicroscopy 11, 53 (1983).

${ }^{37}$ R. Penn and P. Apell, J. Phys. C 16, 5729 (1983).

${ }^{38}$ T. L. Ferrell and P. M. Echenique, Phys. Rev. Lett. 55, 1526 (1985).

${ }^{39}$ A. Howie and R. H. Milne, Ultramicroscopy 18, 427 (1985).

${ }^{40}$ A. H. R. García-Molina, A. Gras-Martí, and R. H. Ritchie, J. Phys. C 18, 5335 (1985).

${ }^{41}$ P. M. Echenique, J. Bausells, and A. Rivacoba, Phys. Rev. B 35, 1521 (1987).

${ }^{42}$ N. Zabala and P. M. Echenique, Ultramicroscopy 32, 327 (1990).

${ }^{43}$ J. Aizpurua, A. Rivacoba, and S. P. Apell, Phys. Rev. B 54, 2901 (1996).

${ }^{44}$ F. J. García de Abajo and J. Aizpurua, Phys. Rev. B 56, 15873 (1997).

${ }^{45}$ J. Aizpurua, A. Howie, and F. J. García de Abajo, Phys. Rev. B 60, 11149 (1999).

${ }^{46}$ Y. T. Chu, R. J. Warmack, R. H. Ritchie, W. Little, R. S. Becker, and T. L. Ferrell, Part. Accel. 16, 13 (1984).

${ }^{47}$ A. Rivacoba, P. Apell, and N. Zabala, Nucl. Instrum. Methods Phys. Res. B 96, 465 (1995).

${ }^{48}$ G. F. Bertsch, H. Esbensen, and B. W. Reed, Phys. Rev. B 58, 14031 (1998).

${ }^{49}$ N. Zabala, E. Ogando, A. Rivacoba, and F. J. García de Abajo, Phys. Rev. B 64, 205410 (2001).

${ }^{50}$ Y. N. Wang and Z. L. Miskovic, Phys. Rev. A 66, 042904 (2002).

${ }^{51}$ J. L. Gervasoni and N. R. Arista, Phys. Rev. B 68, 235302 (2003).

${ }^{52}$ A. A. Lucas, L. Henrard, and P. Lambin, Phys. Rev. B 49, 2888 (1994).

${ }^{53}$ A. Rivacoba and F. J. García de Abajo, Phys. Rev. B 67, 085414 (2003).

${ }^{54}$ D. De Zutter and D. De Vleeschauwer, J. Appl. Phys. 59, 4146 (1986).
${ }^{55}$ N. Zabala, A. G. Pattantyus-Abraham, A. Rivacoba, F. J. García de Abajo, and M. O. Wolf, Phys. Rev. B 68, 245407 (2003).

${ }^{56}$ F. J. García de Abajo, N. Zabala, A. Rivacoba, A. G. PattantyusAbraham, M. O. Wolf, and P. M. Echenique, Phys. Rev. Lett. 91, 143902 (2003).

${ }^{57}$ R. H. Ritchie and A. L. Marusak, Surf. Sci. 4, 234 (1966).

${ }^{58}$ D. L. Johnson and P. R. Rimbey, Phys. Rev. B 14, 2398 (1976).

${ }^{59}$ P. M. Echenique, R. H. Ritchie, N. Barberán, and J. Inkson, Phys. Rev. B 23, 6486 (1981).

${ }^{60}$ R. Fuchs and R. G. Barrera, Phys. Rev. B 24, 2940 (1981).

${ }^{61}$ P. M. Echenique, Philos. Mag. B 52, L9 (1985).

${ }^{62}$ R. Fuchs and K. L. Kliewer, Phys. Rev. 185, 905 (1969).

${ }^{63}$ B. B. Dasgupta and R. Fuchs, Phys. Rev. B 24, 554 (1981).

${ }^{64}$ R. Fuchs and F. Claro, Phys. Rev. B 35, 3722 (1987).

${ }^{65}$ R. Rojas, F. Claro, and R. Fuchs, Phys. Rev. B 37, 6799 (1988).

${ }^{66}$ P. J. Feibelman, Prog. Surf. Sci. 12, 287 (1982).

${ }^{67}$ P. Gies and R. R. Gerhardts, Phys. Rev. B 36, 4422 (1987).

${ }^{68}$ D. E. Beck, Phys. Rev. B 35, 7325 (1987).

${ }^{69}$ K. Kempa, A. Liebsch, and W. L. Schaich, Phys. Rev. B 38, 12645 (1988).

${ }^{70}$ V. Kresin, Phys. Rev. B 39, 3042 (1989).

${ }^{71}$ S. DasSarma and W. Y. Lai, Phys. Rev. B 32, 1401 (1985).

${ }^{72}$ J. Wang and J. P. Leburton, Phys. Rev. B 41, 7846 (1990).

${ }^{73}$ G. Gumbs and A. Balassis, Phys. Rev. B 71, 235410 (2005).

${ }^{74}$ G. Gumbs, A. Balassis, and P. Fekete, Phys. Rev. B 73, 075411 (2006).

${ }^{75}$ Y. H. Tu, C. M. Kwei, Y. C. Li, and C. J. Tung, Phys. Rev. B 74, 045403 (2006).

${ }^{76}$ A. Rubio, L. C. Balbás, and J. A. Alonso, Phys. Rev. B 46, 4891 (1992).

${ }^{77}$ E. Prodan, P. Nordlander, and N. J. Halas, Chem. Phys. Lett. 368, 94 (2003).

${ }^{78}$ R. Chang and P. T. Leung, Phys. Rev. B 73, 125438 (2006).

${ }^{79}$ M. Quijada, R. Díez-Muiño, and P. M. Echenique, Nanotechnology 16, S176 (2005).

${ }^{80}$ J. Yan, Z. Yuan, and S. Gao, Phys. Rev. Lett. 98, 216602 (2007).

${ }^{81}$ V. M. Silkin, A. García-Lekue, J. M. Pitarke, E. V. Chulkov, E. Zaremba, and P. M. Echenique, Europhys. Lett. 66, 260 (2004).

${ }^{82}$ B. Diaconescu et al., Nature (London) 448, 57 (2007).

${ }^{83}$ J. M. Pitarke, V. M. Silkin, E. V. Chulkov, and P. M. Echenique, Rep. Prog. Phys. 70, 1 (2007).

${ }^{84}$ A. Rivacoba, N. Zabala, and J. Aizpurua, Prog. Surf. Sci. 65, 1 (2000).

${ }^{85}$ L. Hedin, B. I. Lundqvist, and S. Lundqvist, Solid State Commun. 5, 237 (1967).

${ }^{86}$ M. Abramowitz and I. A. Stegun, Handbook of Mathematical Functions (Dover, New York, 1964).

${ }^{87}$ I. S. Gradshteyn and I. M. Ryzhik, Table of Integrals, Series and Products (Academic, New York, 1980).

${ }^{88}$ J. C. Ashley and L. C. Emerson, Surf. Sci. 41, 615 (1974).

${ }^{89}$ J. D. Jackson, Classical Electrodynamics (Wiley, New York, 1980). 